\title{
Therapeutic antibody targeting microtubule-binding domain prevents neuronal internalization of extracellular tau via masking neuron surface proteoglycans
}

\author{
Petronela Weisová ${ }^{*}$ (D), Ondrej Cehlár ${ }^{1}$, Rostislav Škrabana ${ }^{1}$, Monika Žllková1, Peter Filipčík', Branislav Kováčech', \\ Michal Prčina', Lubica Wojčiaková', Lubica Fialová', Tomáš Smolek', Eva Kontseková', Norbert Žilka and \\ Michal Novák ${ }^{2}$
}

\begin{abstract}
Pathologically altered tau protein is a common denominator of neurodegenerative disorders including Alzheimer's disease (AD) and other tauopathies. Therefore, promising immunotherapeutic approaches target and eliminate extracellular pathogenic tau species, which are thought to be responsible for seeding and propagation of tau pathology. Tau isoforms in misfolded states can propagate disease pathology in a template-dependent manner, proposed to be mediated by the release and internalization of extracellular tau. Monoclonal antibody DC8E8, binding four highly homologous and independent epitopes in microtubule-binding domain (MTBD) of diseased tau, inhibits tau-tau interaction, discriminates between healthy and pathologically truncated tau and reduces tau pathology in animal model in vivo. Here, we show that DC8E8 antibody acts via extracellular mechanism and does not influence viability and physiological functions of neurons. Importantly, in vitro functional assays showed that DC8E8 recognises pathogenic tau proteins of different size and origin, and potently blocks their entry into neurons. Next, we examined the mechanisms by which mouse antibody DC8E8 and its humanized version AX004 effectively block the neuronal internalization of extracellular AD tau species. We determined a novel mode of action of a therapeutic candidate antibody, which potently inhibits neuronal internalization of AD tau species by masking of epitopes present in MTBD important for interaction with neuron surface Heparan Sulfate Proteoglycans (HSPGs). We show that interference of tau-heparane sulfate interaction with DC8E8 antibody via steric hindrance represents an efficient and important therapeutic approach halting tau propagation.
\end{abstract}

Keywords: Alzheimer's disease, Tau, Immunotherapy, Mechanism of action, Spreading, Heparan sulfate

\section{Introduction}

Alzheimer's disease (AD) and related neurodegenerative disorders are the leading cause of dementia worldwide and their prevalence is rapidly increasing. Despite intensive research and pharmaceutical progress, understanding the trigger or defining the cause(s) of Alzheimer's disease are still among the main scientific challenges of neurodegeneration research. Neurofibrillary pathology formed by abnormal forms of microtubule-associated

\footnotetext{
* Correspondence: weisova@axon-neuroscience.eu

${ }^{1}$ Department of Neuroimmunology, Axon Neuroscience R\&D Services SE,

Dvořákovo nábrežie 10, Bratislava, Slovak Republic

Full list of author information is available at the end of the article
}

protein tau has been recently emerged as a dominant factor in $A D$ pathogenesis, and has been identified as the main correlate of cognitive decline in $\mathrm{AD}$ and other tauopathies. Current research of tauopathies focuses on neurofibrillary lesions, a common pathological hallmark composed of deposits of tau protein in aggregated form [58]. While physiologically functional tau is an unfolded monomeric protein responsible for microtubule stabilisation, neuronal outgrowth and regulation of organelle transport in axons [61], pathological forms of tau which include truncated monomers, oligomers and higher order aggregates are toxic to neurons [77]. We and others have found that misfolded tau protein is directly

(c) The Author(s). 2019 Open Access This article is distributed under the terms of the Creative Commons Attribution 4.0 International License (http://creativecommons.org/licenses/by/4.0/), which permits unrestricted use, distribution, and reproduction in any medium, provided you give appropriate credit to the original author(s) and the source, provide a link to the Creative Commons license, and indicate if changes were made. The Creative Commons Public Domain Dedication waiver (http://creativecommons.org/publicdomain/zero/1.0/) applies to the data made available in this article, unless otherwise stated. 
responsible for synaptic impairment, neuroinflammation, blood brain barrier abnormalities and cognitive or motor deficits in rodent animal models [5, 39, 43, 52, 67, 105, 107].

Progressive development of tau pathology in AD is dependent on transmissibility of aggregated tau protein throughout the brain, thus leading to a characteristic pattern of spatiotemporal/hierarchical spreading [17]. The tau pathology manifest in the trans-entorhinal cortex and spreads in stereotypical manner via neuroanatomically connected brain areas [8, 12, 40, 55]. Aggregated and misfolded tau protein variants have the ability to propagate disease pathology in a templatedependent manner, which is termed as seeding [65]. Interestingly, misfolded tau aggregates from Alzheimer's disease (AD) and other tauopathy brains may vary in their seeding and propagation propensity $[22,35,47,53$, 76]. The propagation of tau throughout the CNS occurs via cell to cell transfer of misfolded tau forms, released by neurons to extracellular milieu which subsequently enter healthy adjoined neurons and induce further fibrillization [9, 26, 28, 34, 48, 95]. Therefore, promising therapeutic intervention consists of targeting extracellular pathogenic tau variants, which are the mediators of seeding and spreading of tau pathology. Indeed, functional cellular assays testing antibodies mapping various epitopes on tau protein are currently used for screening for the best therapeutic antibody candidate based on their ability to block tau internalization and seeding [15, $68,88,102]$, with antibodies targeting predominantly mid-domain of tau showing the maximum potency. Sulfated heparan proteglycans (HPSGs) on the cell membrane are required for the uptake and secretion of misfolded tau species [27, 37, 46, 74, 84]. Therefore, therapeutic interventions with capability to block tauHSPGs interactions and thus preventing spread of tau pathology in the brain may be an important factor potentially contributing to desired clinical benefit.

Previously, we have developed a unique anti-tau antibody DC8E8 interacting with four highly homologous epitopes present in the microtubule-binding domain (MTBD) of tau protein, with its binding mode ensuring the effective blocking in $\beta$-structure formation [49]. Additionally, DC8E8 antibody exhibited higher affinity for pathologically truncated tau over physiological tau and effectively protected the brain from neurofibrillary pathology in vivo [49].

In the present study, we provide a detailed mechanistic insight into the therapeutic mode of action of DC8E8 antibody at neuronal level. Our data revealed predominant extracellular mechanism of action of DC8E8 and its strong ability to recognise all misfolded tau aggregates varying in size, conformation and origin, ensured by its binding epitope to tau. Importantly, DC8E8 proved to be a potent suppressor of internalization and seeding of tau aggregates into primary neurons, thus effectively intercepting spreading of tau to neihgbouring neurons. Binding of tau aggregates to sulfated heparan proteoglycans (HSPGs) on neuronal surface requires heparin binding motifs that are localised in close vicinity to DC8E8 binding epitopes. Our results suggest that intercepting the binding of tau seeds to heparan sulfate surface proteins, with DC8E8 antibody targeting tau in the microtubule-binding domain is an effective immunotherapeutic strategy.

\section{Material and methods \\ Antibodies}

Monoclonal antibody AT8 recognizing a phosphorylated tau pS202-pT205-pS208 and tau monoclonal antibody HT7 recognising the epitope 159-163 of human tau protein were purchased from Thermo Fisher Scientific, antibody DC190 mapping tau 368-376 was purified from hybridoma supernatant and conjugated to horse radish peroxidase. Antibodies DC8E8 [49] and DC25 (epitope 347353) were affinity purified from serum-free hybridoma supernatant. Indiferent monoclonal antibody DC51 (IgG1 isotype) was used as unrelated control (specifically binds to surface glycoprotein of rabies virus) [56]. Monoclonal antitau antibodies AX004/IgG1 and AX004/IgG4, humanized version of anti-tau monoclonal antibody DC8E8 [49] were produced in Expi-CHO cells (Thermo Fisher Cat. No. A29133). The activity of purified antibodies was verified by ELISA, Western blot and immunohistochemistry.

\section{DC8E8 vaccine administration}

DC8E8 vaccine was administered in 2 weeks intervals starting at the age of 6 weeks and continuing until the age of 6 months. Purified antibody DC8E8 prepared in PBS was injected intraperitoneally (1 mg of antibody/per dose $/ 200 \mu \mathrm{l}$ ) into transgenic mice $\mathrm{R} 3 \mathrm{~m} / 4$ expressing human truncated tau (151-391/3R) under the control of the mouse Thy 1 promoter, with tau pathology predominantly located in the brainstem [107]. An additional set of $\mathrm{R} 3 \mathrm{~m} / 4$ animals was injected in the same vaccination scheme (timing and dosing) with unrelated antibody DC51 (anti-rabies IgG1), thus representing the control. Then animals were sacrificed and their brainstems were subjected to immunohistochemical and biochemical quantification of tau pathology. The experiment was carried out in accordance with the Slovak and European Community Guidelines, approved by the State Veterinary and Food Administration of the Slovak Republic.

\section{Quantitative immunohistochemistry of mouse brain tissue samples}

Mouse were anesthetized and perfused intra-cardially for $1 \mathrm{~min}$ with phosphate-buffered saline (PBS), followed by 
3 min perfusion with 4\% paraformaldehyde (PFA) in PBS (4\% PFA, pH 7.2). Brain were transferred for post fixation for $24 \mathrm{~h}$ in $4 \%$ PFA, then transferred to PBS, embedded in paraffin and serially cut using Leica RM 2255 microtome into $8 \mu \mathrm{m}$ thick sagittal brain sections. Immunostaining was performed using the standard immunohistochemistry staining procedure. Briefly, brain sections were treated with $80 \%$ formic acid (40 s) followed by heat pretreatment for $20 \mathrm{~min}$ in antigen retrieval solution (Retrieval 2100, Aptum, Southampton, UK). The sections were blocked with Aptum section block (Aptum, Southampton, UK) followed by incubation with mouse monoclonal primary antibody (Anti -human phospho tau AT8, 1:1000, ThermoScientific, IL, USA) overnight at $4{ }^{\circ} \mathrm{C}$ and were immunostained using the standard avidin-biotin-peroxidase method (Vectastain ABC kit) with VIP as chromogen (VIP kit, Vector Laboratories, Burlingame, CA, USA). After mounting, sections were evaluated using Olympus BX51 microscope. Neurofibrillary tangles were counted in two parallel sections from each mouse brain. Quantification of structures was carried out by investigators blinded to the treatment status of the mice.

Preparation of sarkosyl-insoluble tau protein ( $2 p$ fraction) Brainstems of transgenic animals $\mathrm{R} 3 \mathrm{~m} / 4$ were subjected for biochemical analysis of sarkosyl-insoluble tau proteins. The sarkosyl-insoluble fraction was extracted according to published protocol [32]. Frozen tissues were homogenized for $30 \mathrm{~s}$ in tenfold weight excess of icecold extraction buffer $(20 \mathrm{mM}$ Tris, pH 7.4, $800 \mathrm{mM}$ $\mathrm{NaCl}, 1 \mathrm{mM}$ ethylene glycol tetraacetic acid), $1 \mathrm{mM}$ ethylenediaminetetraacetic acid, $10 \%$ sucrose, containing protease inhibitor (Roche Diagnostics) and phosphatase inhibitor cocktail (Sigma Aldrich). The homogenates were centrifugated at $20,000 \mathrm{~g}$ for $20 \mathrm{~min}$ at $2{ }^{\circ} \mathrm{C}$. The supernatants designed $1 \mathrm{~s}$ were transferred into clean tubes. Next, solid sarkosyl (N-lauroylsarcosine sodium salt; Sigma-Aldrich) was added to the $1 \mathrm{~s}$ supernatant to achieve $1 \%$ concentration and then stirred for $1 \mathrm{~h}$ at room temperature (RT). Samples were then centrifuged at $100,000 \mathrm{~g}$ for $1.5 \mathrm{~h}$ at RT. Pellets (designated $2 \mathrm{p}$ ) were gently rinsed with $1 \mathrm{ml}$ of the sarkosyl extraction buffer and spun at $100,000 \mathrm{~g}$ for $20 \mathrm{~min}$ at RT. The pellets (sarkosyl-insoluble tau fractions) were resuspended in $6 \mathrm{M}$ Guanidine for ELISA or in PBS for Western Blotting analysis to a final volume representing the $1 / 25$ volume of the $1 \mathrm{~s}$ fraction followed by $5 \mathrm{~min}$ sonication and stored at $-20^{\circ} \mathrm{C}$. The sarkosyl-insoluble $2 \mathrm{p}$ tau fractions were also isolated from human $\mathrm{AD}$ brain tissue using the same procedure as described above. Human brain samples (transentorhinal cortex, NFTs rich Braak stage VI, AD sporadic and AD familial with a missense mutation of PSEN1 (Thr116Asn) [85]) were obtained from
Newcastle Brain Bank and Slovak Brain Bank in accordance with ethical approval.

\section{Biochemical quantification of sarkosyl-insoluble tau using sandwich ELISA}

Sarkosyl-insoluble tau fractions (2p) were subjected for biochemical quantification using sandwich ELISA assays (AT8/DC190 for transgenic animal efficacy study; DC25/ DC190 for neuronal internalization in vitro experiments). A 96-well ELISA plate (Nunc Medisorp, Denmark) was coated with $2 \mu \mathrm{g} / \mathrm{ml}$ AT8 antibody (efficacy studies) or DC25 antibody (AD tau neuronal internalization) in PBS overnight $(16-18 \mathrm{~h})$ at $4{ }^{\circ} \mathrm{C}$. The plate was washed five times with PBS buffer supplemented with $0.075 \%$ Tween 20 (PBST) followed by blocking with PBST buffer for $1 \mathrm{~h}$ at RT. Guanidine hydrochloride samples of sarkosyl-insoluble $2 \mathrm{p}$ fractions were diluted 50-fold with PBST buffer (in duplicates). For standard curves, recombinant pathogenic tau 151$391 / 4 R$ (in vitro phosphorylated, affinity purified) was used, in 2 fold dilution steps in PBST buffer. The plate with tau standard and sarkosyl-insoluble tau fractions was incubated $90 \mathrm{~min}$ at $37^{\circ} \mathrm{C}$, followed by washing with PBST for five times. As detection antibody DC190-HRP at 1: 15000 dilution in $\operatorname{PBST}(0,3 \mu \mathrm{g} \mathrm{Ab} / \mathrm{ml})$ was used and incubated for $60 \mathrm{~min}$ at $37^{\circ} \mathrm{C}$, followed by washing with PBST for five times. Next, substrate Colorburst Blue (TMB/peroxide substrate, ready to use; Alerchek USA) was added to the plate and incubated for $20 \mathrm{~min}$ in dark. The reaction was stopped by adding of $0.25 \mathrm{M}$ $\mathrm{H}_{2} \mathrm{SO}_{4}$. The $450 \mathrm{~nm}$ absorbance was measured and plotted against the protein concentration of tau 151-391/4R standard. Concentrations of AT8-positive (efficacy study) or DC25-positive tau (neuronal internalization) in samples was obtained based on extrapolation from the standard calibration curve.

\section{Expression, purification and fibrillization of recombinant tau protein}

Truncated tau 297-391/4R, dGAE (t-tau; numbering according to the longest human tau isoform 2N4R) was expressed in Escherichia coli strain BL21(DE3) (SigmaAldrich, St. Louise, Missouri, United States) from a pET17 expression vector and purified from bacterial lysates as described previously [16], except the anion-exchange chromatography step was omitted and size-exclusion chromatography was performed in PBS-argon (137 mM $\mathrm{NaCl}, 2.7 \mathrm{mM} \mathrm{KCl}, 10 \mathrm{mM}$ Na2HPO4, 2 mM KH2PO4, pH 7.4) (AppliChem GmbH, Darmstadt, Germany). Purified tau protein was stored in PBS-argon in working aliquots at $-70{ }^{\circ} \mathrm{C}$. The purity of tau protein was subsequently verified by gradient SDS gel electrophoresis (5 to $20 \%$ gel), Coomassie blue staining and Western blot analysis with DC25 antibody (AXON Neuroscience SE, 
Bratislava, Slovakia), recognizing residues 347-354 of the longest human tau isoform $2 \mathrm{~N} 4 \mathrm{R}$ ). In vitro fibrillisation of recombinant truncated tau protein $(100 \mu \mathrm{M})$ was carried out using heparin (Sigma-Aldrich, St. Louis, Missouri, United States) as an inducer at a concentration $240 \mu \mathrm{M}$ tau $+60 \mu \mathrm{M}$ heparin in PBS $(137 \mathrm{mM} \mathrm{NaCl}, 2.7$ $\mathrm{mM} \mathrm{KCl,} 10 \mathrm{mM}$ Na2HPO4, $2 \mathrm{mM} \mathrm{KH2PO4,} \mathrm{pH} \mathrm{7.4).}$ The reaction was performed for $24 \mathrm{~h}$ at $37^{\circ} \mathrm{C}$. After incubation, tau aggregates were sonicated for $2 \mathrm{~min}$ at $20 \%$ power output using an MS72 probe of a Bandelin Sonopuls Sonifier (Bandelin, Berlin, Germany). Subsequently, $1 \mu \mathrm{M}$ aliquots were stored at $-70^{\circ} \mathrm{C}$. The oligomerization of the tau protein was verified by nonreducing SDS-PAGE gel electrophoresis and quantitative thioflavin $\mathrm{T}$ (ThT) fluorescence spectroscopy with excitation at $450 \mathrm{~nm}$ and emission at $510 \mathrm{~nm}$.

\section{Fluorescence labelling}

The fluorescently tagged tau protein (after fibrillization) was prepared by labelling with Alexa Fluor ${ }^{\mathrm{TM}}$ dyes (Invitrogen, Carlsbad, California, United States) according to the manufacturer's recommendations. The labeling was carried out at $\mathrm{pH} 6.5$ for preferential labeling of $\mathrm{N}$ terminus. Succinimidyl esters of Alexa Fluor ${ }^{\mathrm{TM}} 488$ and Alexa Fluor $^{\mathrm{TM}}$ 594 (ThermoFisher) were dissolved in anhydrous DMSO (Molecular probes) and mixed with tau proteins (in PBS) in 5:1 $\mathrm{M}$ ratio. The mixture was incubated at RT for $1 \mathrm{~h}$ with $400 \mathrm{rpm}$ shaking. The unreacted dye was subsequently separated from the labeled tau protein using Zeba $7 \mathrm{k}$ MWCO desalting spin columns (Thermo Fisher Scientific) equilibrated with PBS. Labeled truncated tau (297-391/4R) and human brainderived AD Tau (2p sarkosyl-insoluble fraction) were incubated at $37^{\circ} \mathrm{C}$ with $700 \mathrm{rpm}$ shaking for 2 days. The size distribution was monitored using DLS with DynaProNanoStar (Wyatt technologies). The sample was centrifuged for $5 \mathrm{~min}$ at $5000 \mathrm{~g}$ prior to DLS measurement.

\section{Dynamic light scattering (DLS)}

Tau protein samples $(10 \mu \mathrm{l})$ were centrifuged at 5000 $\mathrm{g}$ for $5 \mathrm{~min}$ at $25^{\circ} \mathrm{C}$, transferred into a $4 \mu \mathrm{l}$ disposable cuvette (Wyatt Technology) and measured in a DynaproNanoStar instrument controlled by Dynamics software v. 7.7.0.125 (Wyatt Technology). Measurements were performed in 1 second acquisition time averaged 10-times except for truncated tau dGAE monomer, which was measured using $10 \mathrm{~s}$ acquisition time. Data from at least five individual measurements of dynamic light scattering (DLS) per sample were evaluated by the Dynamics software v. 7.8.0.26. To cull the acquisitions influenced by dust or irregular particles, an automatic filtering of autocorrelation functions was applied with an individual limit for baseline threshold and maximal allowed sum-of-squares (SOS) error for cumulants fit. After filtering, at least $65 \%$ of original data remained for analysis. To determine the size distribution of protein preparations, DLS autocorrelation data were subjected to a regularization analysis by Dynals algorithm. Final graphs were prepared in Prism 6 software (GraphPad).

\section{Infrared spectroscopy}

Infrared spectra were collected on ThermoScientific Nicolet iS50R Research FTIR Spectrometer equipped with a DTGS detector (Thermo Fisher Scientific). The instrument and sampling accessory were continuously purged with water and $\mathrm{CO}_{2}$ free air. One $\mu \mathrm{l}$ of sample in PBS was loaded into a ConcentratIR2 Multiple Reflection ATR (Harrick Scientific Products) adopting a silicon element with a nominal incident angle of $30^{\circ}$ and eleven reflections. The sampling plate was sealed and the sample drop dried under flow of dry air. After vanishing of liquid water absorption bands, the flow of dry air was stopped and 32 scans were collected at $4 \mathrm{~cm}^{-1}$ resolution within a spectral range of $650-4000 \mathrm{~cm}^{-1}$ wavenumbers. Spectra were collected using zero-filling factor 2, Happ-Genzel apodization, Mertz phase correction, aperture 160, samples gain 4 , optical velocity 0.4747 $\mathrm{cm} . \mathrm{s}^{-1}$. Reference spectra were recorded under identical conditions with empty ATR sampling plate and were subtracted from the protein-sample spectra. Spectra were further baseline-corrected and processed with ATR advanced correction as implemented in OMNIC software v. 9 (Thermo Fisher Scientific).

\section{Kinetics of truncated tau (297-391/4R) aggregation - Thioflavin T (ThioT) fluorescence spectrometry}

Detection of tau filaments assembly-aggregation of recombinant tau protein was monitored by ThT fluorescence. $40 \mu \mathrm{l}$ of $350 \mu \mathrm{M}$ truncated-tau 297-391 (dGAE) with $20 \mu \mathrm{M}$ ThioflavinT (Sigma) was incubated in black solid polystyrene 384 wells Greiner BioOne plate in the absence and presence of heparin (tau-heparin concentration ratio 4:1). The fluorescence was measured using the Fluoroskan Ascent FL (Labsystems) every $10 \mathrm{~min}$ with excitation at $450 \mathrm{~nm}$ and emission at $510 \mathrm{~nm}$. The plate was shaken at $720 \mathrm{rpm}$ and incubated at $37^{\circ} \mathrm{C}$. The aggregation kinetics was monitored for 4 days.

\section{Immunoprecipitation of native, sarkosyl-insoluble AD tau with DC8E8 antibody}

The sarkosyl resistant $2 \mathrm{p}$ tau fraction was isolated from $1 \mathrm{~g}$ of AD human brain sample using the procedure outlined above for mice brain tissue. The brain was examined by immunohistochemistry and tau pathology found to correspond to Braak stage VI. The $2 \mathrm{p}$ pellet fraction was re-suspended in $1 \mathrm{ml}$ of PBS (supplemented with 50 $\mathrm{mM} \mathrm{NaF}, 1 \mathrm{mM} \mathrm{Na} 3 \mathrm{VO} 4$ and the cocktail of protease 
inhibitors Complete ${ }^{\bullet}$ without EDTA (Roche)) by sonication for $2 \mathrm{~min}$ on ice using a Bandelin Sonopuls HD2200/UW2200 equipped with a MS72 probe, at 20\% duty cycle with the output set at 20\% (Bandelin Electronic, Germany). The resulting suspension was split into two $500 \mu \mathrm{l}$ portions and each portion received $25 \mu \mathrm{g}$ of one of two purified antibodies: either DC8E8 or a control antibody DC51. The suspensions were incubated with the antibodies with head-over-tail rotation at $6{ }^{\circ} \mathrm{C}$ for $2 \mathrm{~h}$. In order to isolate the antibody-disease tau complexes, $50 \mu \mathrm{l}$ of the $50 \%$ suspension of Protein G Mag Sepharose beads (GE Healthcare), equilibrated in PBS+ $0.01 \%$ Igepal CA-630 (SIGMA), were added to each of the two mixtures of sarkosyl-insoluble AD tau and antibodies. The immunoprecipitation mixtures were further incubated at $6{ }^{\circ} \mathrm{C}$ for $1 \mathrm{~h}$. The beads in each of the incubation mixtures with bound antibody-tau complexes were harvested using magnet and washed three times with $150 \mu \mathrm{l}$ of PBS (supplemented with $50 \mathrm{mM} \mathrm{NaF}, 1$ mM Na3VO4, 0.02\% IGEPAL CA-630 (SIGMA) and the cocktail of protease inhibitors Complete ${ }^{\circ}$ without EDTA (Roche)) and once with $150 \mu \mathrm{l}$ of PBS only. The bound antibody complexes were eluted from the beads by three consecutive 5-min incubations in $100 \mu \mathrm{l}$ of $200 \mathrm{mM}$ formic acid $\mathrm{pH}$ 2.7. The eluates were pooled, lyophilized, the proteins dissolved in SDS-PAGE sample loading buffer, separated on 12\% SDS-PAGE gels, transferred onto nitrocellulose membranes and the tau proteins detected by incubation with the pan tau antibody DC25 conjugated to HRP (Kementec, Denmark). The blots were developed with a SuperSignal West Pico Chemiluminescent Substrate system (Pierce, U.S.A) and the signals detected using a LAS3000 imaging system (FUII Photo Film Co., Japan).

\section{Cortico-hippocampal neurons, viability, viral transduction and cellular physiological functions}

Rodent cortico-hippocampal neurons were prepared and cultured as described, with minor modifications [10]. Neocortes were isolated from pregnant female mice C57BL6N on embryonic day 16-18 using 10\% Ketamidor and 10\% Xylariem as lethal anesthesia. Brains of embryos were dissected out, placed in ice-cold sterile L-15 medium free of L-glutamine (PAA) and meninges were removed. Cerebral cortices with hippocampi were isolaed, chopped into small pieces and incubated with $0.25 \%$ Trypsin-EDTA for $10-15$ min at $37^{\circ} \mathrm{C}$. After incubation, trypsinisation was inhibited by adding media with inactivated serum. The cells were dissociated by gentle pipetting with subsequent centrifugation at $300 \mathrm{~g}$ for $5 \mathrm{~min}$. Cortico-hippocampal neurons were triturated with glass Pasteur pippete in fresh plating media (DMEM supplemented with 10\% (v/v) fetal calf serum, 2 $\mathrm{mML}$-glutamine and $100 \mathrm{units} / \mathrm{mL}$ penicillin/streptomycin (all from Life Technologies Invitrogen, Carlsbad, California, United States). Cells were plated onto Poly-D-lysine coated 6 well plates or glass Nunc Lab-Tek Chambers (Thermo Fisher Scientific) at a density of $2.10^{5}$ cells $/ \mathrm{cm}^{2}$ (unless indicated otherwise) and and cultivated at $37{ }^{\circ} \mathrm{C}, 5 \% \mathrm{CO} 2$ in a water-saturated atmosphere. After $24 \mathrm{~h}$ plating media was exchanged for Neurobasal Media containing 2\% B27, 2 $\mathrm{mML}$-glutamine and gentamycin $10 \mu \mathrm{g} / \mathrm{mL}$. Experiments were carried out after $2 \pm 5$ days in vitro (DIV). Transfection of primary neurons with fluorescently labelled antibody DC8E8 Alexa Fluor $546(30 \mu \mathrm{g})$ was performed with MaxCyte Flow Electroporation ${ }^{\mathrm{m}}$ Technology according to the manufacters instructions and cells were examined for viability/nuclear morphology (Hoechst 33258 Sigma, 1 rg/ml; Trypan blue Sigma) and physiological functions (ENLITEN ATP Assay System Bioluminescence Detection kit; Promega) 24h later. Alternatively, primary neurons 1-2 DIV were transduced with virus containing human truncated tau - AAV9-hSynapsin1-tau (151-391)-P2AmCherry WPRE (Vector Biolabs). Two to three days after AAV viral transduction $\left(9.1 \times 10^{13} \mathrm{GC} / \mathrm{ml}\right)$ neurons were examined for t-tau (151-391/4R) protein expression with immunocytochemistry and western blotting. All procedures involving animal work were in accordance with ethical standards and approval from the State Veterinary and Food Committee of Slovak Republic and the number of sacrifised animals recorded.

\section{Neuronal tau internalization assay}

Truncated tau (297-391/4R) and human brain-derived $\mathrm{AD}$ tau (transentorhinal cortex from two sporadic $\mathrm{AD}$ patients and one familial AD case [85], both NFT rich Braak stage VI) fluorescently labelled with Alexa Fluor 488 or Alexa Fluor 594 were diluted in neuronal conditioned media at the concentration corresponding to $100-200 \mathrm{nM}$ for monomeric tau protein in combination with the following antibodies: unrelated control antibody DC51 [56], mouse monoclonal DC8E8 (mDC8E8), humanized DC8E8 (AX004/IgG1; AX004/IgG4) and their isotype controls IgG1/IgG4 (BioLegend), all of concentration of $1 \mu \mathrm{M}$ and incubated for $30 \mathrm{~min}$ at $37^{\circ} \mathrm{C}$. Preformed tau-antibody complexes were added to neurons for $24 \mathrm{~h}$. For experiments where heparin was used (Tinzaparin sodium, Sigma-Aldrich, which is a LMWH), fluorescently labelled tau aggregates were preincubated with heparin $(5 \mu \mathrm{M})$ for $30 \mathrm{~min}$ and heparin-AD tau complexes were applied to neurons for $16-20 \mathrm{~h}$. Neurons were washed three times with pre-warmed PBS, followed by mild trypsinisation (0.06\% trypsin-EDTA $2-$ $3 \mathrm{~min}$ ) to remove cell surface bound tau and dissociate neurons into single cells. Next, neurons were processed live for flow cytometry measurements to quantify the amount of fluorescently labelled internalized tau (BD 
LSRFortessa ${ }^{\text {tx }}$ II cell analyzer). Neurons were gated using forward and side scatter to remove cellular debris. Measurements were recorded as mean fluorescent intensity of Alexa Fluor 488 or Alexa Fluor 594. Alternatively, neurons grown on glass Nunc Lab-Tek Chambers (Thermo Fisher Scientific) were imaged for fluorescence AF488 labelled tau protein (excitation at $488 \mathrm{~nm}$ and emission at $525 \mathrm{~nm}$ ) with a $20 \times$ objective LSM 710 confocal microscopy and examined for intracellular localisation by fluorescent labelling of lysosomes with fluorescent dye LysoTracker $(75 \mathrm{nM}, 45 \mathrm{~min}$ Thermo Fisher Scientific). Typically, experiments were performed in triplicates from minimum of three independent experiments and 10000 cells for each well were quantified by flow cytometry. Data were analysed using Prism Software (GraphPad).

\section{Immunocytochemistry}

Rodent cortico-hippocampal neurons were cultured on cover glass pre-coated with Poly-D-lysine at a density of $1 \times 10^{6}$ cells per milliliter and cultivated for $48 \mathrm{~h}$. Neurons were treated with fluorescently labelled (Alexa Fluor 488 or Fluor 594) sarkosyl-insoluble AD tau (2p, $100 \mathrm{nM})$ only or in combination with control and DC8E8/AX004 antibody $(1 \mu \mathrm{M})$ and cultivated for $20 \mathrm{~h}$ in conditioned Neurobasal media at $37^{\circ} \mathrm{C}, 5 \% \mathrm{CO}$. Next day neurons were washed with pre-warmed PBS and mild trypsin (0,06\% trypsin-EDTA, $3 \mathrm{~min})$, fixed with 4\% PFA-PHEM, pH 6.9 (60 mM PIPES, $25 \mathrm{mM}$ HEPES, $10 \mathrm{mM}$ EGTA, $2 \mathrm{mM} \mathrm{MgCl}_{2}$, PFA) for $12 \mathrm{~min}$. Neurons were permeabilized with $0.1 \%$ Triton X100 in PBS (PBS-T) and blocked with 5\% BSA in TBS-T. The cells were then incubated with anti-heparan sulfate antibody (10E4, AMSBIO 1:100) for $1 \mathrm{~h}$ or, in a different experiment with HT7 antibody (epitope against human tau, MN1000, Thermo Fisher Scientific 1:500), washed and incubated with secondary antibody goat anti-mouse Alexa Fluor 488 (Invitrogen). The samples were mounted in FluoroshieldTM medium with DAPI (Sigma-Aldrich). Images were captured by LSM 710 confocal microscope (Zeiss, Jena, Germany).

\section{Western blot analysis of fibrillized and sarkosyl-insoluble} tau

Samples of fibrillized truncated tau 297-391/4R (1 $\mu \mathrm{g})$ and human-derived AD tau fractions $(6 \mu \mathrm{l})$ from sarkosyl isolations (2p, $1 \mathrm{~s}$ and $2 \mathrm{~s}$ ) were mixed with SDS sample loading buffer and heated at $95^{\circ} \mathrm{C}$ for $5 \mathrm{~min}$. Each sample $(6 \mu \mathrm{l})$ was then loaded onto $5-20 \%$ gradient SDS polyacrylamide gels and electrophoresed in a Trisglycine-SDS buffer system for $40 \mathrm{~min}$ at $25 \mathrm{~mA}$. Proteins were transferred to nitrocellulose membrane and, after blocking in 5\% fat-free dry milk in PBS for $1 \mathrm{~h}$ at room temperature, the membrane was incubated for $1 \mathrm{~h}$ with pan-tau mAb DC25 and with therapeutic antibody DC8E8. After washes, HRP-conjugated goat anti-mouse Ig (Dako Denmark) diluted 1:3,000 in PBS was used as a secondary antibody. Blots were washed and developed using ECL chemiluminescence detection (GE Healthcare), detected with SuperSignal West Pico Chemiluminescent Substrate (Pierce Biotechnology), and imaged using a FujiFilm LAS-3000 imaging system (Fuji).

\section{Statistical analysis}

Data are presented as means \pm SEM. To compare two groups, the Mann-Whitney $U$ test or an unpaired $t$-test was applied. For statistical comparison of more groups, one-way analysis of variance ANOVA and post hoc Tukey's test were used using Prism software v. 7 (GraphPad Software, Inc., San Diego, USA). Differences were considered significant at the level of $p<0.05$.

\section{Results}

Antibody targeting microtubule binding region (MTBR) provides neuroprotection against tau pathology in transgenic animals

A growing body of recent experiments revealed that the antibody epitope rather than its affinity to pathological tau proteins is an important factor in mediating therapeutic efficacy $[15,88]$. Previously, we generated a murine monoclonal DC8E8 antibody with the strong potential to abolish pathological tau-tau interaction by targeting four separate epitopes localized in human tau protein microtubule binding regions MTBR1-4 [49]. In the biosensor SPR analysis, antibody DC8E8 exhibits nanomolar afinity with nearly an order of magnitude stronger binding to pathological truncated tau protein than to physiological full-length tau [44]. In order to determine the therapeutic potency of DC8E8 antibody in vivo, we utilized transgenic mice expressing pathogenic non-mutated human truncated tau (151-391/3R) with the ability to induce neurofibrillary pathology similar to human NFTs located predominantly in the brainstem $[73,107]$. Transgenic mice were treated with mDC8E8 or unrelated control antibody DC51 of the same isotype (IgG1) biweekly ( $40 \mathrm{mg} / \mathrm{kg}$ ) for 5 months. In line with our previous results [49], we confirmed the strong ability of this therapeutic antibody to reduce the development of neurofibrillary pathology measured here with independent method of sarkosyl-insoluble tau-specific ELISA (Fig. 1 a). A significant suppression of the development of tau pathology in the brainstem of transgenic mice treated with DC8E8 in contrast to animals treated with control antibody DC51 was measured (with $\sim 70 \%$ decrease in the amount of sarkosyl-insoluble tau). Quantitative histopathological examination of brains from transgenic animals treated with DC8E8 antibody revealed reduced accumulation of AT8 positive pretangle 


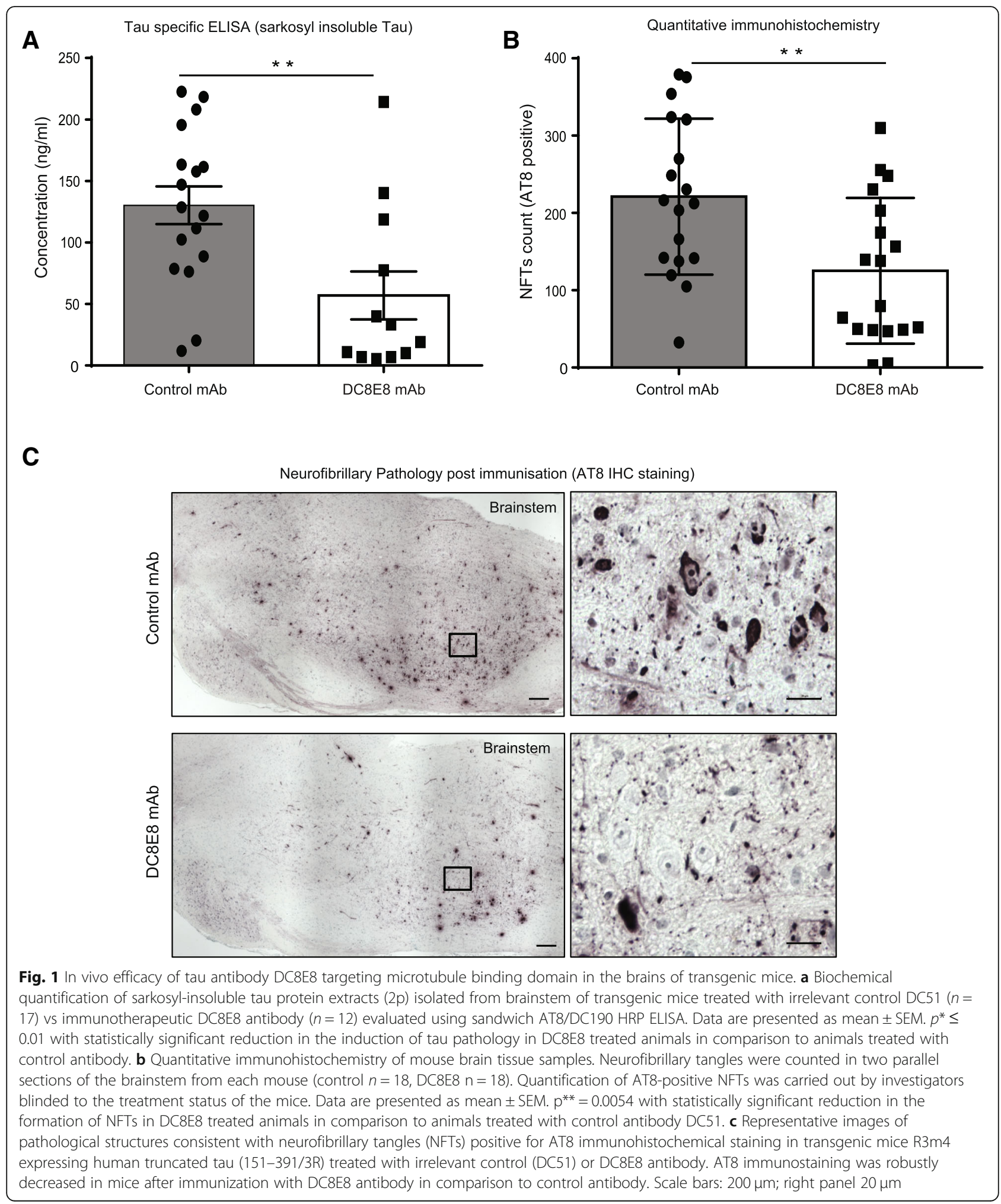

and tangle bearing neurons when compared to mice treated with control antibody (Fig. 1 b, 1c), and supported the high efficacious effect of DC8E8. Biochemical and histopathological measurements, thus demonstrated a robust therapeutic effect of DC8E8 antibody targeting four separate conformational tau epitopes in MTBR, as shown by a strong in vivo supression of tau pathology development in the brains of transgenic mice. Similarly, 
AADvac1 active vaccine, currently in clinical trials, that targets one of DC8E8 epitopes and elicits production of antibodies with the same tau binding properties as DC8E8 [73], proved to be efficacious with the capability to reduce the development of tau pathology in transgenic animals to similar extent $[49,50]$.

\section{DC8E8 antibody acts via extracellular mechanism and does not influence viability of neuronal cells}

Previous pre-clinical evidences from $A \beta$ and tau immunotherapies demonstrated that therapeutic antibodies can cross blood-brain barrier and accumulate in the brain to mediate their effects $[2,7,30,31,75,81]$. To gain a mechanistic insight into the therapeutic mode of action of DC8E8 antibody at neuronal level, we first tested (in ex vivo conditions) whether antibody DC8E8 is internalized into neurons containing diseased tau proteins to mediate their clearance. Murine corticohippocampal primary neurons derived from embryonic day 17.5 pups were infected with adeno-associated virus containing pathogenic truncated tau (t-tau, 151-391/3R) $[24,107]$ linked to red fluorescent protein mCherry. AAV-mediated transduction of pathogenic tau was evaluated by immunocytochemistry using pan tau antibody (DC190) in neurons cultured for 5 DIV. Tau protein expression was confirmed by robust colocalization of AAV-transduced neurons positive for mCherry and tau antibody (DC190) staining in cell bodies and neurites, as well as by immunoblotting with a band of size specific for truncated tau (25 kDa), (Fig. $2 \mathrm{a}, \mathrm{b})$. To monitor the uptake of DC8E8 antibody into tau diseased neurons, we performed immunocytochemistry to examine the intracellular localisation of DC8E8 (Fig. 2 c). Interestingly, we observed the intracellular presence of DC8E8 only in neurons with compromised plasma membrane integrity (Fig. 2 c). To confirm that DC8E8 antibody is not internalized in tau diseased neuronal cells, we utilized an additional model of neuroblastoma cells SH-SY5Y expressing t-tau (151-391/4R). Confocal live cell imaging with fluorescently labelled DC8E8 antibody (red) added into the cell culture media of tau expressing cells showed comparable results. We found that antibody was only detected intracellularly in tau expressing cells with condensed nuclei, not in live neuronal cells. Taken together, our findings suggest that the predominant therapeutic effect of DC8E8 antibody is extracellular. This finding is in a line with previous reports of other immunotherapies targeting tau protein $[44,102]$.

We next investigated whether the therapeutic candidate DC8E8 has any harmful effects on neurons in vitro. Since the antibody is not uptaken into neurons spontaneously, cells were transfected with fluorescently labelled DC8E8 antibody (Alexa Fluor 546) using MaxCyte electroporation. Already at $2 \mathrm{~h}$ after electroporation DC8E8 antibody decorated microtubules inside neuroblastoma cells SH-SY5Y (Fig. 3 a), thus confirming that the antibody molecules are functional and retained their ability to bind tau. We then examined the effect of DC8E8 antibody on neuronal viability at $24 \mathrm{~h}$ after electroporation using Hoechst staining (Fig. 3 b, c). Viability, here expressed as percentage of non-condensed nuclei in neurons with intracellular localisation of fluorescent antibody, was not affected when comparing neurons transfected with DC8E8 or control antibody (Fig. 3 b, c). We also assessed physiological functions of neurons at $24 \mathrm{~h}$ after electroporation of DC8E8 into cells. We did not detect any significant change in ATP levels between neuronal populations electroporated with control or DC8E8 antibody (Fig. 3 d). Neuronal viability was also not affected when DC8E8 antibody was present extracellularly by its addition to the surrounding culture media (data not shown). In summary, these data confirmed that DC8E8 antibody did not interfere with viability or physiological functions of neurons, thus strongly supporting its safety properties also at a cellular level.

\section{Extracellular pathogenic tau species of various size and conformation are internalized by neurons in concentration and time-dependent manner}

Tau protein in Alzheimer's disease can form multiple different variants and species via the pathogenic process of misfolding [19]. The presence of pathogenic tau proteins actively released from diseased neurons in vitro and detected also in interstitial fluid (ISF) and CSF, clearly demonstrated the importance of extracellular tau species in the propagation of tau pathology $[11,62,87$, 96-100]. The spread of extracellular tau protein species with its initial step of tau neuronal entry $[26,95]$ has been shown to be regulated by tau quaternary structure $[45,74]$. Formation of tau fibrillar filamentous species with cross- $\beta$-structure/core and morphology similar to NFTs can be potentiated in vitro $[4,6,60]$. The truncated tau protein (dGAE, 297-391), which encompasses the PHF-core region, assembles into PHF-like fibrils through the repeat domain as revealed by cryo-EM study $[4,23,25]$. Therefore, to investigate whether primary neurons can spontaneously internalize relevant pathogenic tau variants, we used the in vitro fibrillized form of recombinant truncated 95-amino acid tau fragment (297-391/4R) [4, 82, 94]. To cover the whole spectrum of truncated oligomerized and otherwise modified tau species (tauons) present in $\mathrm{AD}$ brain, we also prepared sarkosyl-insoluble fractions (2p) from sporadic and familial human AD brains (Braak stage VI). AD tau aggregates contain a compact core rich in the cross-beta structure [25]; using Thioflavin T (ThT) fluorescence, infrared spectroscopy and dynamic light scattering, we characterized the assembly of in vitro pre-formed 


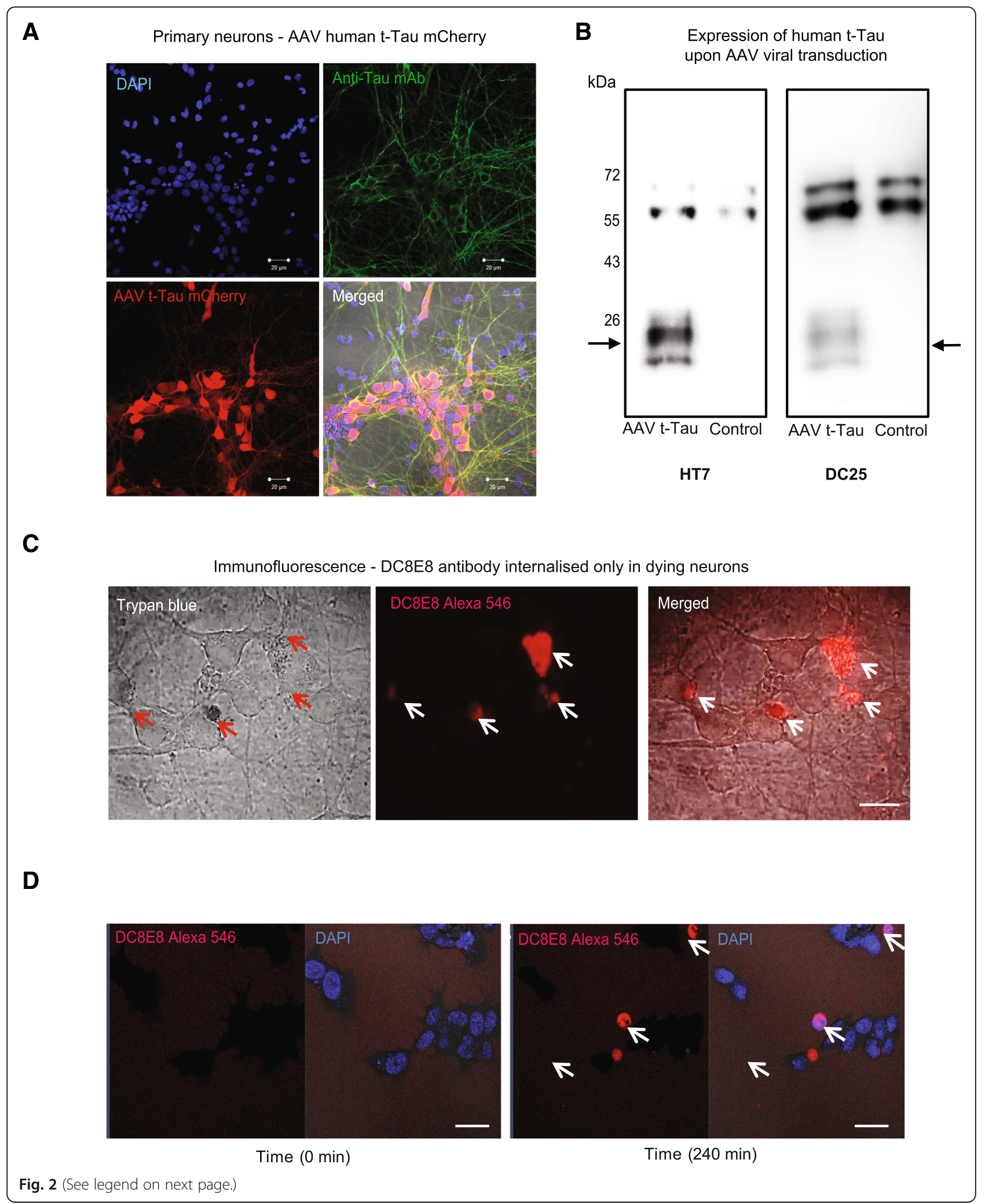


(See figure on previous page.)

Fig. 2 Monoclonal antibody DC8E8 requires no uptake into primary neuronal cells with pathogenic truncated tau. a Characterization of primary cortico-hippocampal neurons transduced with pathogenic AAV human truncated tau linked to mCherry (red). Forty eight hours after AAV viral transduction neurons were examined for truncated tau expression (151-391/4R) by immunocytochemistry with anti tau antibody (DC190 recognising epitope 368-376, green). Merged image confirmed substantial co-localization between the AAV transduced t-tau and additional pan tau staining. $\mathbf{b}$ Three days after AAV transduction primary neurons were screened for t-tau protein expression by Western Blot utilizing 2 different anti tau antibodies (HT7; DC25 recognising 347-353). SDS-PAGE and immunoblotting in both cases show expression of the $25 \mathrm{kDa}$ human truncated tau protein in neuronal cells as indicated (arrows). c Primary neurons cultured on glass with confirmed AAV transduced t-tau protein were examined for viability with trypan blue. Subsequently, neurons were subjected to addition of DC8E8 antibody. Immunocytochemical labelling of DC8E8 showed that therapeutic antibody was detected and localized only in trypan blue positive neurons (dying neurons) as indicated by arrows. $\mathbf{d}$ Human neuroblastoma cells SHY5Y expressing human truncated tau protein (151-391/3R), were cultured on glass Labtec dishes and stained live for condensed nuclei (Hoechst $333581 \mu \mathrm{g} / \mathrm{mL}$ ). Next, cells were subjected to an addition of fluorescently labelled humanized version of DC8E8/AX004 Alexa Fluor 546 (red) in media $(1 \mu \mathrm{M} \sim 168 \mu \mathrm{g} / \mathrm{mL})$ and monitored over time with LSM 710 confocal microscopy for up to $4 \mathrm{~h}$. AX004 antibody was not internalized in neuronal cells with t-tau expression, only in dying cells with condensed nuclei (Hoechst) as indicated with arrows. Experiments were repeated 3 times with similar results obtained. Scale bars, $10 \mu \mathrm{m}$

recombinant truncated tau fibrils in terms of internal structure and size (Fig. 4a-d). Development of ThT fluorescence during assembly reaction indicates increasing content of beta structure, which eventually reached a plateau (Fig. 4a). FTIR spectroscopy of truncated tau fibrils (297-391/4R), compared with those of monomeric truncated tau, revealed nearly complete disappearance of a broad absorption maximum characteristic of disordered monomer $\left(1642 \mathrm{~cm}^{-1}\right)$ and creation of a sharp maximum at $1629 \mathrm{~cm}^{-1}$, which can be ascribed to the beta-sheet component of amide I region [79] (Fig. 4b). Characterization of all three different preparations of tau (fibrillized truncated tau, AD sporadic and AD familial tau) by DLS confirmed the presence of a wide spectrum of tau pathogenic species varying in size (with $20-100 \mathrm{~nm}$ radii) (Fig. 4c) and conformations (Fig. 4b, c, d).

Finally, we analysed if therapeutic candidate antibody DC8E8 can recognise the set of prepared tau species (recombinant truncated tau, human derived AD sporadic tau and AD familial sarkosyl-insoluble tau). Indeed, we confirmed the ability of DC8E8 antibody to capture the entire range of pathogenic tau species of different sizes (tau aggregation intermediates, fibrils and both low- and high-molecular-weight oligomers, multimers comprising all stages of tau protein oligomerization) and origin (Fig. 4d, e). Recognition of a complete panel of pathogenic tau protein oligomers and aggregates is an important prerequisite for their effective neutralization by a candidate therapeutic antibody. Therefore, we have also examined DC8E8 binding to sarkosyl-insoluble high-molecular-weight AD tau extract that had been immunoprecipitated after a gentle sonication, maintaining a native status of tau oligomers. We have compared western blot profile of the $2 \mathrm{p}$ sarkosyl extract of the AD brain with the DC8E8 immunoprecipitated material of the same, using a pan tau, repeat-region specific monoclonal antibody DC25 (tau 347-353) [106]. DC8E8 captured all tau species present in the extract, whereas an antibody with an unrelated specificity DC51 did not capture any tau-reactive material (Fig. 4f). Therefore, in addition to binding monomeric pathological tau proteins [49], DC8E8 also recognizes a full spectrum of high-molecular weight pathological tau.

To ensure that the prepared species can act as tau seeds and their size does not exceed what neurons are able to uptake, the aggregates were sonicated before administration to cell media. We next incubated fluorescently labelled human brain-derived sarkosyl insoluble tau (2p) with murine primary neurons at two increasing concentrations. As examined by live cell confocal imaging (Fig. 5a, b), extracellular AD tau species were readily internalized in primary neurons and were trafficked to lysosomes which futher confirmed their intracellular localisation (Fig. 5b). However, not all the up-taken tau proteins were engulfed in the lysosomes, part of them were present in the "free" cytoplasmic fraction. We suggest that this fraction of tau could serve as a seed for the aggregation of endogenous tau and further propagation of tau pathology. We found that sarkosyl-insoluble $\mathrm{AD}$ tau species were internalized in primary neurons in a concentration-dependent manner which is in accordance with previous reports using tau P301S variant [21]. Moreover, neuronal internalization of fibrillized truncated tau and $\mathrm{AD}$ tau evaluated by different independent methods (flow cytometry, tau specific ELISA, immunocytochemical colocalisation of internalized $\mathrm{AD}$ tau with human tau antibody HT7 and Western Blot), resulted in a statistically significantly increased neuronal uptake of these pathogenic tau species over time (Fig. 5e, f, Additional file 1: Figure S1a, b). Thus, disease relevant tau species in our experiments, covering the whole range of tau variants of different size and origin (Fig. 4b-d) are readily internalized into primary neurons in vitro (Fig. 5 a - e; Additional file 1: Figure S1 a, b). 


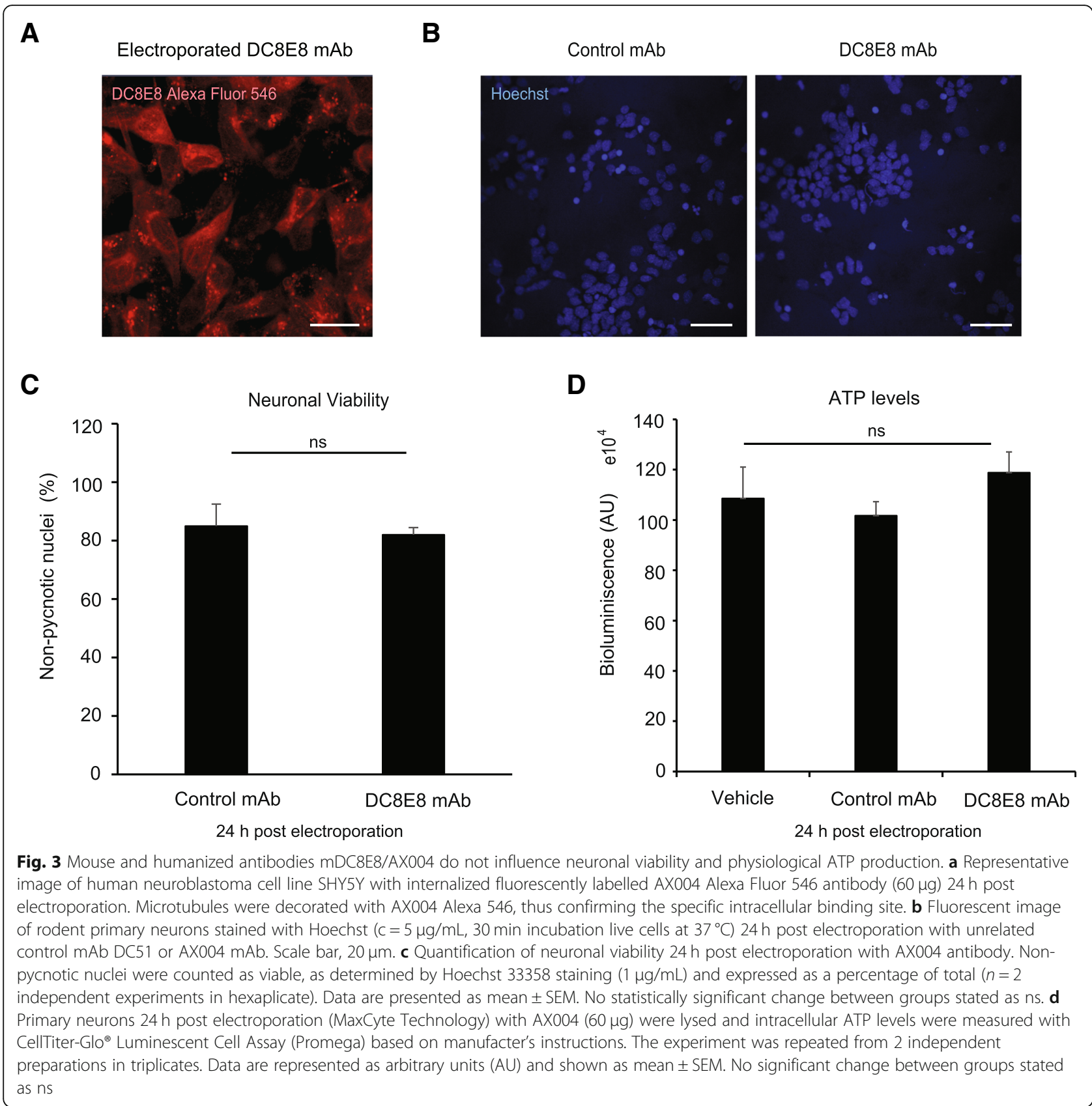

\section{Therapeutic antibody DC8E8 prevents neuronal} internalization of disease-relevant $A D$ tau variants

Recently, in vitro functional assays of tau neuronal uptake and seeding have been used to screen for the best candidate antibody to ensure maximal therapeutic efficacy $[14,15,68,88]$. In these studies, mostly antibodies targeting the mid domain of tau showed the highest capacity to block tau seeding in various in vitro and in vivo models in direct comparison to $\mathrm{N}$ - or C-terminal targeting antibodies $[15,68,88]$. Monoclonal antibody DC8E8 binds to MTBR at four highly homologous sites [49] and can recognise various forms of pathogenic tau (Fig. $4 \mathrm{~d}$, e). DC8E8 therapeutic antibody is not internalized into tau diseased neurons (Fig. 2) and predominantly mediates its mechanism of action extracellularly. Therefore, we next investigated whether DC8E8 and its humanized version (AX004/IgG1), can also capture and neutralize various extracellular pathological tau variants and their neuronal internalization (the initial step of seeding). To do so, we tested the potency of DC8E8 antibody to reduce the uptake of extracellular diseased AD tau into primary neurons. We examined the amount of fluorescently labelled $\mathrm{AD}$ tau internalized to neurons in the presence of control versus DC8E8 antibodies using 


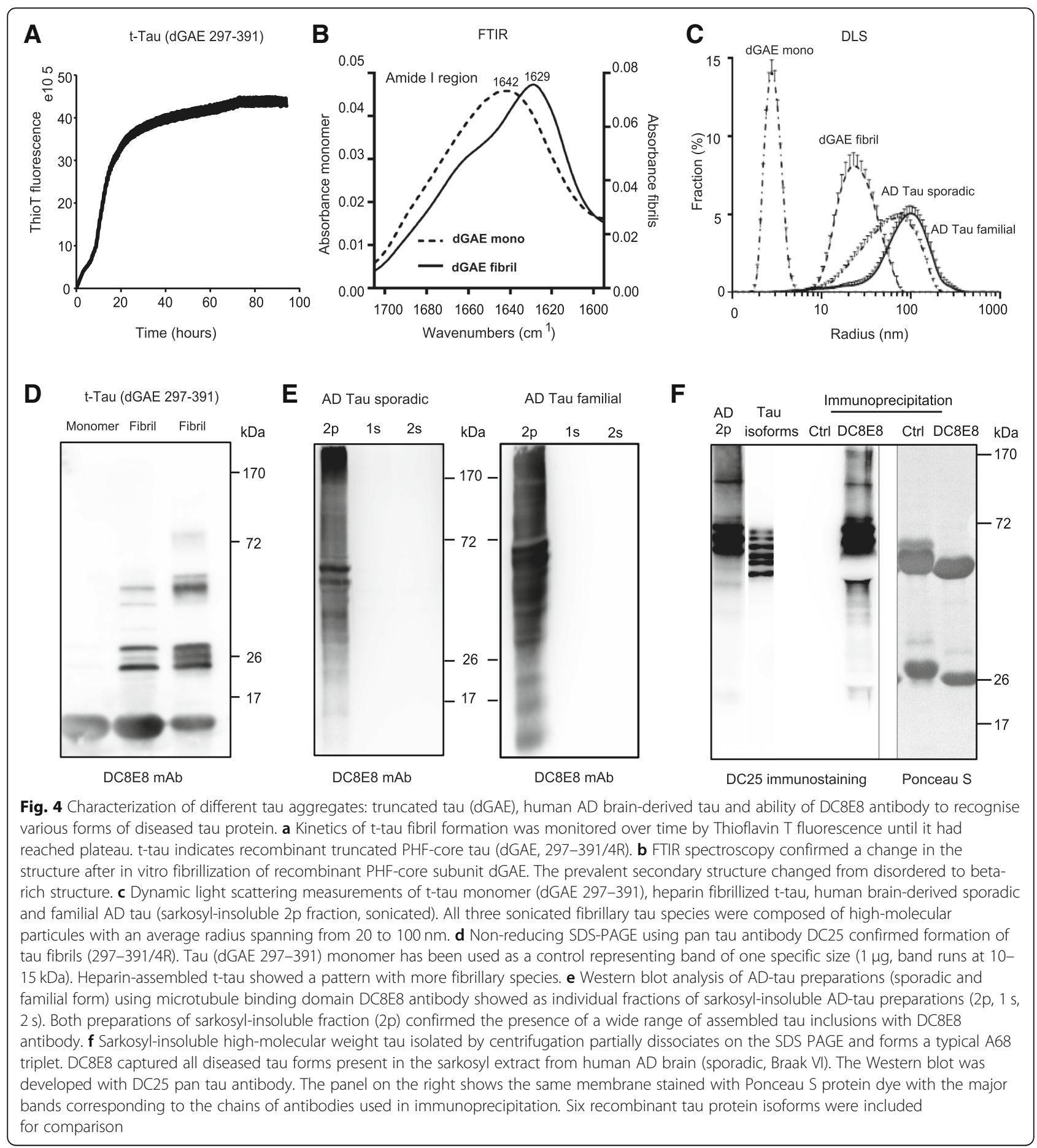

confocal microscopy in combination with flow cytometry and ELISA. Indeed, AD tau fluorescent punctae accumulation within neurons was statistically significantly reduced in the presence of DC8E8 antibody, with larger fluorescent aggregates present outside the cells in media (Fig. 6 a). Presumably, AD tau in the presence of DC8E8 antibody formed complexes that were primarily localized outside the neurons (Fig. 6 a). Under these conditions,
DC8E8 antibody potently blocked the uptake of sporadic sarkosyl-insoluble AD tau already at $6 \mathrm{~h}$ after addition to the culture media with a more pronounced effect after $24 \mathrm{~h}$ of incubation (Fig. $6 \mathrm{c}$ ).

Previous reports and our proteomic data (not shown) suggest the existence of heterogeneous pool of tau variants present in Alzheimers disease brain comprised predominantly of various truncated AD-tau species $[35,44$, 


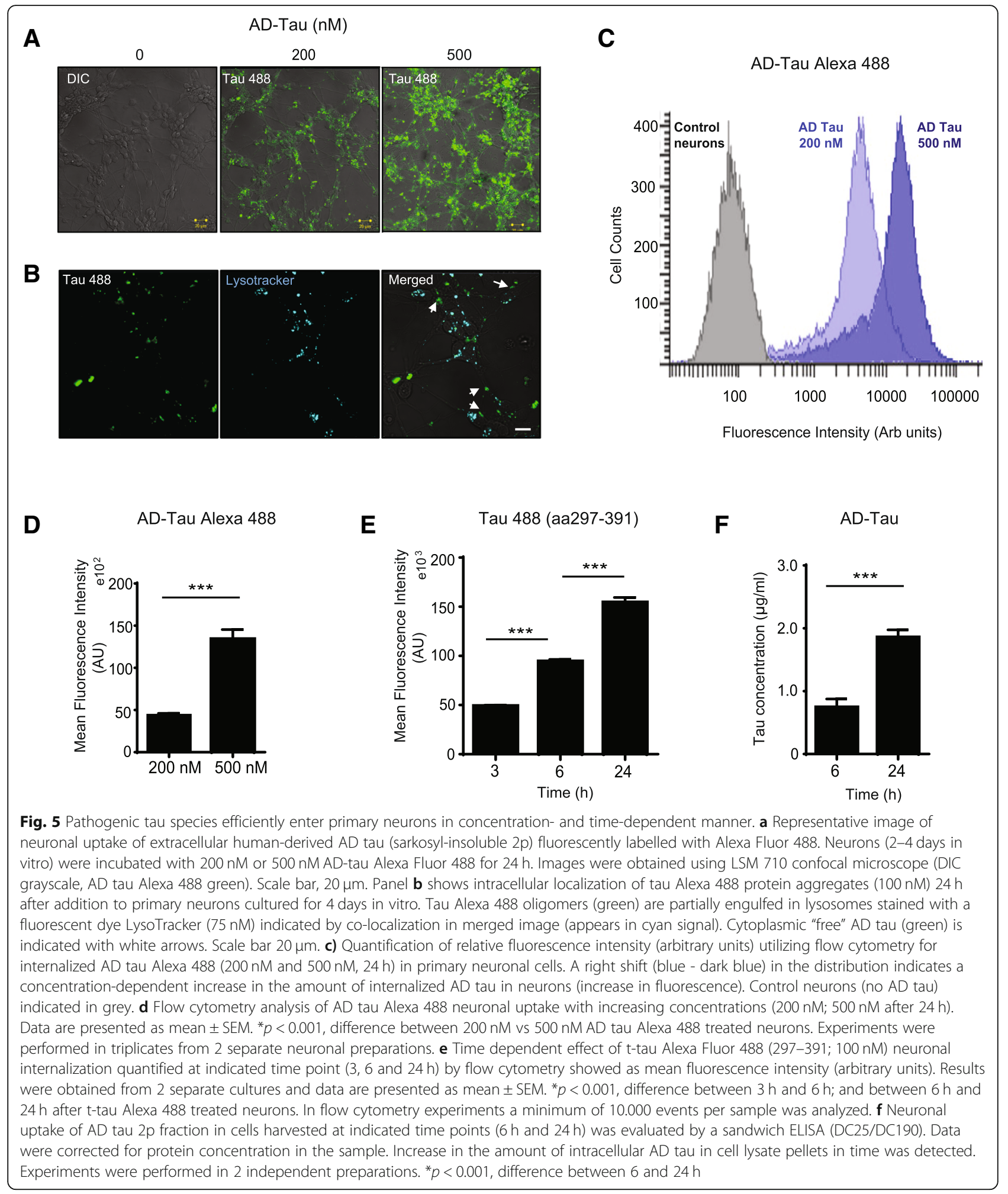

$51,63,104]$. Therefore, to gain a more complex insight we analysed whether DC8E8 antibody can prevent internalization of different relevant pathogenic tau variants present in AD human brain (PHF-core truncated tau,
AD sporadic and AD familiar tau). DC8E8 antibody proved to be a strong potent blocker of tau neuronal uptake for all tau species examined (Fig. $6 \mathrm{~d}$ ). This is a direct evidence that DC8E8 antibody recognises a whole 


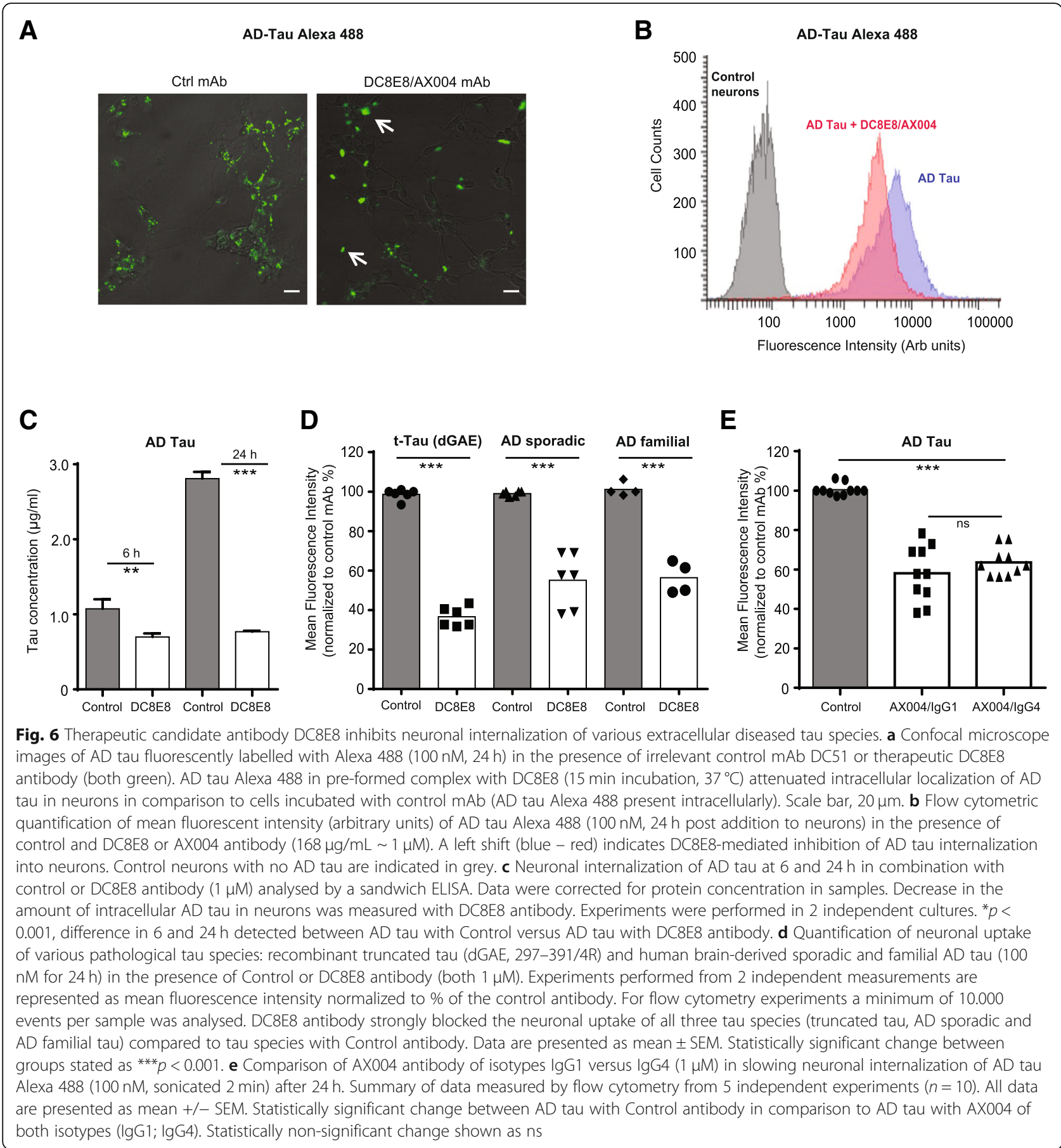

set of pathogenic tau variants (Fig. 4) and is concominantly capable of capturing the majority of extracellular tau by forming complexes with them, and thus preventing their neuronal internalization.

Ultimately, we investigated whether the antibody isotype is actively involved in mediating the blocking effect of $\mathrm{AD}$ tau uptake into neurons. For this purpose, we used humanized version of DC8E8 antibody (AX004) of two different isotypes, AX004/IgG1 or
AX004/IgG4, as these two isotypes have been used for all tau-targeted immunotherapies in clinical development since 2013 [71]. We found that both isotypes of AX004 antibody exhibited a pronounced inhibitory effect on the internalization of AD tau into neurons (approx. by 50\%, Fig. 6 e). Notably, both AX004 isotypes, IgG1 and IgG4 attenuated AD tau neuronal uptake to a similar extent with no statistically significant difference between them (Fig. 6 e). 


\section{Antibody DC8E8 potently abrogates entry of human derived $A D$ tau into neurons via masking tau recognition sites to heparan sulfate proteoglycans (HSPGs)}

An important hallmark of $\mathrm{AD}$ and related tauopathies is the formation of various pathogenic tau variants with strong ability to spread to neuro-anatomically connected regions in the brain [17]. This process involves the release of proteinaceous seeds into the extracellular space and their uptake by neighbouring cells where they induce fibrillization of intracellular tau $[26,95]$. Recent data firmly established the critical role of sulfated heparan proteoglycans in the internalization and release of tau from neurons depending on the aggregated state of tau and its quarternary structure [21, 37, 46, 74, 101]. Pathogenic tau protein variants of specific size and conformation contain several heparin binding motifs predominantly in the microtubule-binding repeats domain (MTBR) important for neuronal internalization [27, 37, 80, 103]. DC8E8 is an anti-tau antibody interacting with 4 different epitopes with homologous amino acid sequence, also localised in each of the repeats of MTBR domain [49]. Therefore, we challenged the hypothesis that the formation of a complex between therapeutic antibody and pathogenic AD tau masks the HSPG binding sites, thus preventing its neuronal entry. Initially, we examined the involvement of HSPGs in sarkosylinsoluble $\mathrm{AD}$ tau in neuronal internalization process. As shown above (Fig. 5 b), the intra-cellular localization of tau has been supported by its colocalization with lysosomes. Here, we detected colocalisation of numerous punctae of $\mathrm{AD}$ tau amyloid fibrills with heparan sulfate present on the surface of neurons (Fig. 7 a). Conversely, when therapeutic DC8E8 antibody was present in the culture media, AD-tau fibrils were not internalized in neurons and no co-localization with heparan sulfate was detected (Fig. 7b). It has been shown by others, that heparin can efficiently block the interaction of tau with HSPGs [37, 103]. Indeed, in the presence of heparin $(5 \mu \mathrm{M}) \mathrm{AD}$ tau failed to bind to neurons and its internalization was potently inhibited (Fig. 7c). The effect of therapeutic antibody DC8E8 or heparin alone on blocking of neuronal AD tau uptake were comparable. We speculate that the slightly higher efficiency of blocking with heparin alone over DC8E8 can be mediated by the presence of other amyloid protein aggregates present in crude sarkosyl-insoluble AD tau fraction. Most importantly, no statistically significant additive effect was observed when neurons where treated with heparin alone or with heparin in combination with DC8E8 antibody (Fig. 7c).

Based on our previous structural data of DC8E8 antibody binding epitopes localised in MTBR (R1-R4) and, embracing the conserved sequence motif HxPGGG present four times on tau protein, we propose the mechanism of how DC8E8 can block AD tau neuronal internalization (Fig. 8 a, b). Multiple HSPGs binding sequences on tau, predominantly localized in R2 and R3 (residues 274-335) and close to lysine-rich region after R4 repeat domain [27, 49, 80, 103] are all in close proximity to DC8E8 antibody binding epitopes: ${ }^{268} \mathrm{HQPGGG}^{273}$ (R1), ${ }^{299}$ HVPGGG ${ }^{304}$ (R2), ${ }^{330}$ HKPGGG ${ }^{335}$ and ${ }^{362}$ HVPGG $^{367}$ (detailed residue level description of HSPG binding sites from NMR data described on Fig. 8 a). Therefore, formation of a complex between DC8E8 antibody and extracellular AD tau reduces the number of available heparan sulfate binding motifs, which interferes with the mode of HSPG-tau interaction and at the same time inhibits further aggregation. Overall, tau sequence analysis data in combination with the presented biological functional analysis, strongly suggest that the unique characteristics of the epitope of DC8E8 antibody determine its therapeutic mode of action as the ability to effectively block tau beta-structure formation and AD tau neuronal internalization by masking HSPG recognition sites via steric hindrance (Fig. 8 a, b).

\section{Discussion}

Immunotherapy targeting the major proteinaceous pathological lesions currently represents the main approach in the search for an AD modifying treatment. Given its critical role in the pathogenesis of $\mathrm{AD}$, and considering the strong correlation of NFTs pathology with cognitive decline of patients, diseased tau proteins have become a very promising molecular target for disease-modifying immunotherapy of AD. Therefore, we have selected a monoclonal anti-tau antibody DC8E8 with a unique tau binding mode consisting in recognition of four homologous epitopes present in each of the repeats of MTBD [44, 49, 72, 73]. Importantly, tau structural determinants characterized by DC8E8 antibody were utilized also for the development of an active vaccine AADvac1 (currently in the clinical trials), which contains one of the DC8E8 epitopes [73]. Similar to active vaccine AADvac1, passive immunotherapy with DC8E8 antibody confirmed its strong efficacy in prevention of the development of tau pathology $[49,50]$. Serum antibodies generated after vaccination with AADvac1 and DC8E8 antibody target the same epitopes on tau, exhibit a strong preference for pathological tau over fulllength tau [70] and display similar efficacious effects in animals expressing pathogenic truncated tau, indicating that both active and passive immunizations share a common mechanism of action.

Detection of intracellular tau protein extracellularly in interstitial space or CSF in AD patients [41, 97-99] and controversy in studies examining intracellular [13, 33, 81] vs extracellular mode of action of antibodies, shifted most of the current research to screen for antibodies 


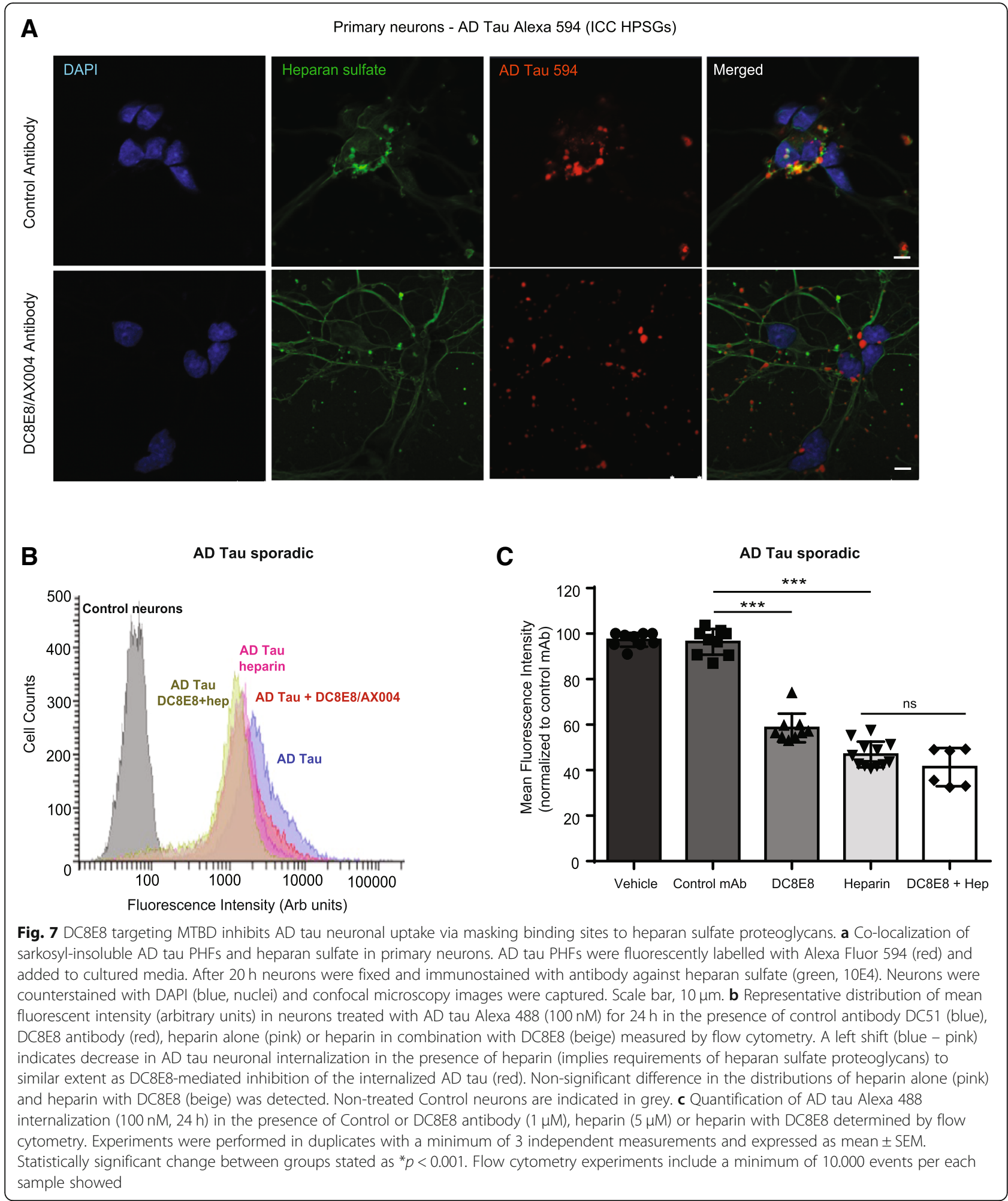

targeting predominantly extracellular tau species [36]. Using ex vivo neuronal culture models we were able to show that DC8E8 antibody was not readily internalized in neurons with diseased tau expression even several hours after addition to culture media, suggesting extracellular mechanism of action as the main factor contributing to its efficacy. This is in a line with other preclinical studies where despite profound efficacy antibodies where not internalized into neurons [102]. However, we could not completely exclude the neuronal 


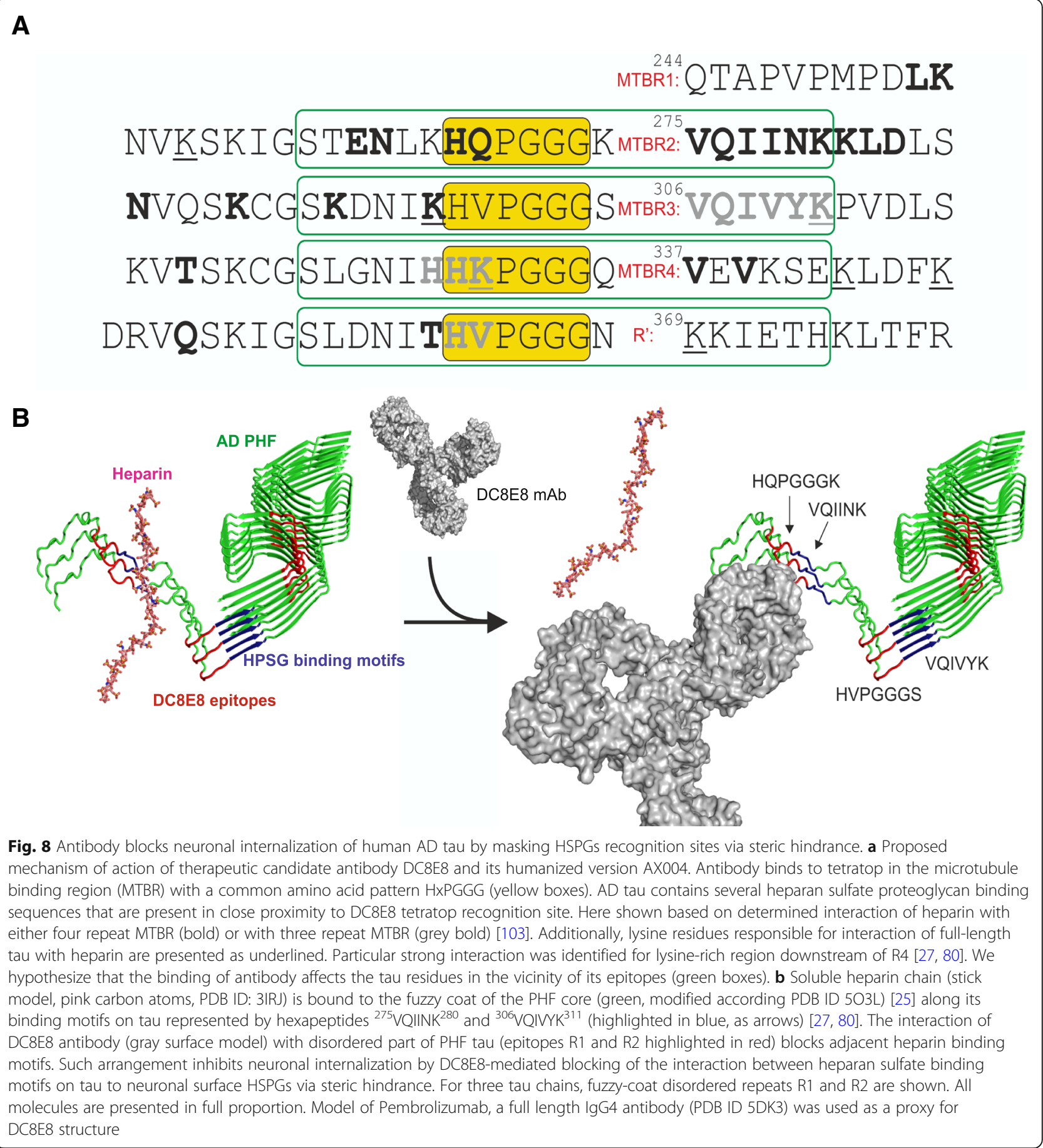

uptake of a small amount of DC8E8 antibody in complex with tau aggregates as found by others [21], which could be either under our detection threshold or due to ex vivo cell culture conditions used which might differ from in vivo conditions. Additionally, Alzheimer's tau protein forms toxic complexes with cellular membranes of primary hippocampal cultures [3], which can then potentially mediate neuronal uptake of the antibody.
Therefore, we examined the binding properties and toxicity of DC8E8 antibody also if present intraneuronally. In ex vivo conditions we electroporated fluorescently labeled DC8E8 antibody into the cytosol of primary neurons. Binding of DC8E8 to microtubule-associated tau proteins was detected very early after electroporation showing that delivered antibody molecules were active. The internalized DC8E8 antibody did not interfere with 
the viability or physiology of neurons even at later time points. Thus, although DC8E8 antibody preferentially binds to pathogenic tau [49], it can interact with physiological tau but obviously does not interfere with its normal function. These results also support our recent findings from the phase I clinical trial of active AADvac1 [70, 73]. The vaccine, which contains one of the DC8E8 epitopes and thus elicits production of DC8E8like antibodies, showed an exceptionally favourable safety profile. Hence, both passive and active DC8E8based immunotherapeutic approaches thus appear safe.

In recent years, tau pathophysiology has been necessarily linked with findings that tau can propagate across neuro-anatomically concected brain regions histologically defined as Braak staging [8, 12]. Trans-neuronal propagation of tau aggregates involves the release of proteopathic seeds into the extracellular space with more free tau entering recipient neurons and initiating further fibrillization. The predominantly extracellular tau protein is thus a readily accesible target for therapeutic antibodies. The efficacy of antibodies depends on the epitope selection and ability to recognise relevant pathogenic tau species responsible for trans-neuronal transmission. In this work we demonstrated the ability of DC8E8 antibody to recognise a heterogeneous pool of pathogenic tau aggregates of various sizes (LMW, HMW), conformations (native status of tau multimers), and origins (recombinant PHF-core truncated tau, sporadic and familial $\mathrm{AD}$ ), making this antibody a suitable therapeutic candidate. These data were confirmed by an independent study showing that an antibody that binds HxPGG motifs, present four times in MTBD, binds all human PHF tau variants [88]. Furthermore, clinical potential of DC8E8 was highlighted by our previous finding where we demonstrated that DC8E8 has a strong binding preference for truncated diseased tau protein (151391/4R) over the physiological full-length tau monomer 2N4R [49], thus overcoming the concerns of harmful effects linked to potential elimination of healthy physiological tau [59].

Another challenge discussed in various studies are the unclear/unknown properties of pathogenic tau species responsible for neuronal toxicity and disease progression in AD brains [19, 42, 64, 86, 87]. Therefore, to supply a wide range of pathogenic tau species naturally present throughout course of Alzheimer disease, we prepared oligomerized and aggregated tau species (sarkosyl-insoluble fraction 2p) from sporadic as well as familial forms of AD (stage Braak VI) [85]. Each fraction was further sonicated to maximize the variability in size and conformations of individual tau species present in $\mathrm{AD}$ brains. Indeed, the strong spreading potency in vivo in animals has been demonstrated also using human-derived seed-competent sarkosyl-insoluble tau fractions as inoculum $[35,42,83,88]$.
Parallel with our previous findings, disease associated tau in post-mortem AD brains, appears to contain large amount of truncated tau fragments $[18,51,69,72,88$, $92,93,104,106]$. Tau fragments truncated at both $\mathrm{N}$ and $\mathrm{C}$ - termini that contain MTBD are more prone to aggregation [51], with tau fragment terminated at Glu 391 in vitro into a cross- $\beta$ core through the repeat domain into PHF $[1,91]$. Thus, as an alternative to the natural truncated tau variants present in $\mathrm{AD}$ brains, here we also tested a recombinant truncated tau fragment dGAE encompassing amino acids 297-391 of full length tau that corresponds to the PHF-core region $[4,25]$. AD-relevant tau variants tested here were readily spontaneously internalized by primary neurons in time and concentration-dependent manner. Similar results have been reported recently, however using other experimental conditions: different tau variants (full-length 2N4R tau fibrils [27], full-length tau with P301S mutation, the cause of familial form of frontotemporal dementia (FTD) rather than $\mathrm{AD}[21])$, or utilizing a non-neuronal cell based seeding assays [88] which requires facilitation of tau seeding with additional protein carriers (lipofectamine) [15].

The central role of misfolded tau aggregates, actively released from diseased neurons to extracellular space, has been demonstrated in the process of trans-neuronal propagation [36, 77]. Therefore, targeting extracellular tau by antibody with the ability to recognise distinct pathogenic tau variants may efficiently abrogate spreading of tau pathology and thus represent an efficient therapeutic strategy. Different sets of cellular in vitro functional assays examining tau neuronal seeding and propagation have been used to identify new immunotherapeutics with a potential maximum efficacy $[15,68$, $88,102]$. Here we tested the ability of a candidate therapeutic antibody DC8E8 to block neuronal internalization of pathogenic tau species, thereby slowing down interneuronal propagation of tau pathology. Indeed, antibody DC8E8 and its humanized version AX004 were both highly effective in neutralization of the initial uptake of various disease-associated tau seeds. Recently, testing different sets of antibodies showed that only antibodies with epitopes localized in mid-region of tau protein, and not at the $\mathrm{N}$-terminus, displayed the highest potential in tau seeds depletion/neutralization [15, 68, 88]. We also confirm here that DC8E8 antibody with its epitope localized in the aggregation-prone MTBR domain has a strong tau uptake neutralization ability, even though the decrease in fluorescence intensity was not complete. We cannot exclude the contribution of other tau-unrelated protein aggregates present in crude sarkosyl-insoluble AD brain material and potentially different sensitivity of our approach when compared to aggregation-biosensor HEK293T cell lines of non-neuronal origin [38]. 
Importantly, another antibody PT83, with highly similar binding pattern as DC8E8, localised in all four repeats of MTBD with xxxPGG motif, showed almost complete inhibition of tau seeding into non-neuronal HEK293 FRET cells over-expressing truncated tau fragment 243-375 [88]. In the same study, another MTBD antibody tested in vivo for neutralization of tau seeding exerted a strong inhibition in transgenic mice. Conversely, firstgeneration therapeutic $\mathrm{N}$-terminal antibodies selected based on their affinity were significantly less active in functional seeding assays and unable to recognise terminaly truncated tau fragments in isolated PHFs [88]. The fact that PHFs from AD brains contain truncated tau variants at either one or both $\mathrm{N}$ - and $\mathrm{C}$ - termini $[18,72$, 106], together with an assumption that $A D$ tau fragments contain at least part of an intact MTBR, predisposes DC8E8 antibody with its unique binding tetratop located throughout this region as a promising therapeutic candidate targeting pathogenic (seed-competent) tau species.

Tau aggregates bind heparan sulfate proteoglycans (HSPGs) on the neuronal surface, promoting tau neuronal uptake and seeding via macropinocytosis, thereby transmitting tau pathology among neurons [37, 74]. Increased amount of sulfated proteoglycans in AD brain [54], colocalisation with neurofibrillary tangles [29], and recently identified involvement of HSPGs in the process of tau neuronal internalization $[27,37,57,74,84,101]$ and tau secretion [46] are indicative of critical role of HSPGs in the transmission of tau pathology among neurons. Neuronal internalization of aggregates by HSPGs has been shown to be dependent on aggregate size, conformation $[27,41,74,84]$ and the presence of specific heparin binding motifs on tau responsible for tau/HSPG interaction $[20,27,80,103]$. Here we demonstrated that DC8E8 antibody can not only recognise various pathogenic tau variants, but has the ability to abrogate tau neuronal entry. Knowing that binding motifs responsible for tau/HSPGs interaction are mainly localized in the same microtubule binding repeat domain as the epitopes of therapeutic candidate antibody DC8E8, we analysed in detail if the formation of a complex between tau aggregates and antibody can potentially mask recognition site on tau important for neuronal internalization process. By employing ADderived tau aggregates which are more likely to be internalized via HSPGs [27], as confirmed here by colocalization of heparan sulfate and internalized AD tau aggregates, we also tested the ability of DC8E8 antibody to inhibit tau neuronal internalization alone or in combination with heparin. These in vitro functional experiments strongly support the hypothesis that the binding of DC8E8 antibody to AD tau most probably masks HSPGs binding motifs on tau, as neuronal entry of tau was abrogated to a similar extent. Interventions targeting the binding of tau aggregates to neuronal sulfate proteoglycans have been suggested as a new therapeutic opportunity [27, 37, 46, 74]. Therefore, to achieved detailed mechanistic insight, we further performed analysis of binding properties of DC8E8 antibody to pathogenic tau protein. Overall, positively charged lysines, arginine, and histidine are the important spots in binding between tau and heparin as determined by NMR titration [66, 80, 103]. Moreover, hexapeptides presents in the second (R2) and third repeat domains (R3) of MTBD on tau: $\left({ }^{275} \mathrm{VQIINK}^{280}\right.$ and $\left.{ }^{306} \mathrm{VQIVYK}^{311}\right)$ defined as aggre gation-prone and associated with PHF-core $[25,78,89$, 90], were also identified as heparin binding motifs [27, 66 , 103]. Notably, the first two epitopes of therapeutic candidate antibody DC8E8 mapping residues 269-273 and 299-304 on tau are localised in close proximity to HSPG binding sequences on tau $[49,72]$. Furthermore, a lysine rich region downstream of $\mathrm{R} 4$ has been also suggested as HSPG binding sequence $[27,80]$ again in proximity to DC8E8 binding to epitope 362-367 present in R4. Similar potential mechanisms of monoclonal anti-tau antibodies decreasing pathology in vivo were also suggested recently [27, 102]. Antibody HJ9.3 recognising epitope 306-320 in MTBR, similar to DC8E8 binding epitope, was found to be the only antibody tested with the unique ability to inhibit internalization of repeat-domain tau fibrils and $\mathrm{AD}$ tau aggregates in primary neurons with proposed potential mechanism of blocking tau/HSPG interaction [27, 48].

Taken together, analysis of tau binding properties of DC8E8 antibody together with the presented functional ex vivo cellular assays strongly indicate that DC8E8 antibody targeting four different epitopes in the MTBR domain of pathogenic tau, has unique therapeutic attributes with several mechanisms of action: 1) DC8E8 binding to tau protein precedes $\beta$-forming motifs which leads to inhibition of aggregation of pathogenic tau [49], 2) can recognise pathological tau variants of distinct sizes and origins (sporadic, familial AD) with preferential binding to pathogenic over physiological tau and most importantly 3) effectively inhibits spreading of tau pathology by interfering with $\mathrm{AD}$ tau neuronal uptake through masking several HSPG recognition sites via steric hindrance. Additionaly, DC8E8 antibody recognises repeat domain of tau even in its oligomerised form (confirmed by inhibition of neuronal uptake of dGAE tau oligomers), thus showing the exceptional ability of DC8E8 to bind to seeding-capable forms of tau. In conclusion, our results highlight a unique therapeutic mechanisms involved in active and passive approaches based on anti-tau DC8E8 antibody and its remarkable tau epitope used for the design of first-in man AADvac1 vaccine currently in Phase 2 human clinical trials [73], thus representing an exciting period in $\mathrm{AD}$ therapy. 


\section{Additional file}

Additional file 1: Figure S1. Human AD brain-derived tau protein internalized in primary neurons. a) Immunocytochemical co-localization of sarkosyl-insoluble AD tau PHFs and HT7 antibody in primary corticohippocampal neurons. AD tau PHFs were fluorescently labelled with Alexa Fluor 488 (green) and added to neuronal cultured media. After $24 \mathrm{~h}$ neurons were fixed and immunostained with antibody against human tau (red, MN1000 ThermoFisher). Neurons were counterstained with DAPI (blue, nuclei) and confocal microscopy images were captured. Scale bar, $10 \mu \mathrm{m}$. b) Western blot analysis of human AD tau (sarkosyl-insoluble $2 p$ ) internalized in primary cortico-hippocampal neurons over time. Experiments were repeated in triplicate with similar pattern obtained. (PDF $923 \mathrm{~kb}$ )

\section{Acknowledgements}

Authors wish to acknowledge L. Belancová, A. Legeňová and M. Marunová for technical assistance. This study was supported by AXON Neuroscience R\&D Services SE.

\section{Authors' contributions}

PW designed and coordinated the study, carried out in vitro experiments and drafted the manuscript. OC performed fluorescent labelling and designed the schematics of interaction of DC8E8 with PHF AD tau. RS conducted biophysical tau characterizations and contributed to manuscript draft. MZ participated in the functional cellular experiments. PF produced humanized antibody AX004. BK carried out immunopulldown assay and contributed to manuscript draft. MP performed flow cytometry. LW prepared AD sarkosyl-insoluble tau species. LF carried out ELISA assays and efficacy study. TS performed quantitative immunohistochemistry. EK, NZ and MN designed therapeutic candidate antibody DC8E8. All authors read and approved the final manuscript.

\section{Competing interests}

The authors declare that they have no competing interests.

\section{Author details}

'Department of Neuroimmunology, Axon Neuroscience R\&D Services SE, Dvořákovo nábrežie 10, Bratislava, Slovak Republic. ${ }^{2}$ Axon Neuroscience SE, Arch. Makariou \& Kalogreon 4, Larnaca, Cyprus.

Received: 15 July 2019 Accepted: 15 July 2019

Published online: 07 August 2019

\section{References}

1. Abraha A et al (2000) C-terminal inhibition of tau assembly in vitro and in Alzheimer's disease. J Cell Sci 113(Pt 21):3737-3745

2. Adolfsson $\mathrm{O}$ et al (2012) An effector-reduced anti-beta-amyloid (Abeta) antibody with unique abeta binding properties promotes neuroprotection and glial engulfment of Abeta. J Neurosci 32(28):9677-9689

3. Ait-Bouziad $\mathrm{N}$ et al (2017) Discovery and characterization of stable and toxic tau/phospholipid oligomeric complexes. Nat Commun 8(1):1678

4. Al-Hilaly YK et al (2017) Alzheimer's disease-like paired helical filament assembly from truncated tau protein is independent of disulfide crosslinking. J Mol Biol 429(23):3650-3665

5. Bennett RE et al (2018) Tau induces blood vessel abnormalities and angiogenesis-related gene expression in P301L transgenic mice and human Alzheimer's disease. Proc Natl Acad Sci U S A 115(6):E1289-E1298

6. Berriman J et al (2003) Tau filaments from human brain and from in vitro assembly of recombinant protein show cross-beta structure. Proc Natl Acad Sci U S A 100(15):9034-9038

7. Bohrmann B et al (2012) Gantenerumab: a novel human anti-Abeta antibody demonstrates sustained cerebral amyloid-beta binding and elicits cell-mediated removal of human amyloid-beta. J Alzheimers Dis 28(1):49-69

8. Braak H, Braak E (1995) Staging of Alzheimer's disease-related neurofibrillary changes. Neurobiol Aging 16(3):271-278 discussion 278-84

9. Calafate $\mathrm{S}$ et al (2015) Synaptic contacts enhance cell-to-cell tau pathology propagation. Cell Rep 11(8):1176-1183

10. Cente M, Mandakova S, Filipcik P (2009) Memantine prevents sensitivity to excitotoxic cell death of rat cortical neurons expressing human truncated tau protein. Cell Mol Neurobiol 29(6-7):945-949
11. Cicognola C et al (2018) Novel tau fragments in cerebrospinal fluid: relation to tangle pathology and cognitive decline in Alzheimer's disease. Acta Neuropathol

12. Clavaguera F et al (2015) Invited review: prion-like transmission and spreading of tau pathology. Neuropathol Appl Neurobiol 41(1):47-58

13. Congdon EE et al (2013) Antibody uptake into neurons occurs primarily via clathrin-dependent Fcgamma receptor endocytosis and is a prerequisite for acute tau protein clearance. J Biol Chem 288(49):35452-35465

14. Congdon EE et al (2016) Affinity of tau antibodies for solubilized pathological tau species but not their immunogen or insoluble tau aggregates predicts in vivo and ex vivo efficacy. Mol Neurodegener 11(1):62

15. Courade JP et al (2018) Epitope determines efficacy of therapeutic anti-tau antibodies in a functional assay with human Alzheimer tau. Acta Neuropathol 136(5):729-745

16. Csokova N et al (2004) Rapid purification of truncated tau proteins: model approach to purification of functionally active fragments of disordered proteins, implication for neurodegenerative diseases. Protein Expr Purif 35(2):366-372

17. de Calignon A et al (2012) Propagation of tau pathology in a model of early Alzheimer's disease. Neuron 73(4):685-697

18. Derisbourg M et al (2015) Role of the tau N-terminal region in microtubule stabilization revealed by new endogenous truncated forms. Sci Rep 5:9659

19. Dujardin S et al (2018) Different tau species lead to heterogeneous tau pathology propagation and misfolding. Acta Neuropathol Commun 6(1):132

20. Elbaum-Garfinkle S, Rhoades E (2012) Identification of an aggregation-prone structure of tau. J Am Chem Soc 134(40):16607-16613

21. Evans LD et al (2018) Extracellular monomeric and aggregated tau efficiently enter human neurons through overlapping but distinct pathways. Cell Rep 22(13):3612-3624

22. Falcon B et al (2015) Conformation determines the seeding potencies of native and recombinant tau aggregates. J Biol Chem 290(2):1049-1065

23. Falcon B et al (2018) Tau filaments from multiple cases of sporadic and inherited Alzheimer's disease adopt a common fold. Acta Neuropathol 136(5):699-708

24. Filipcik $P$ et al (2012) First transgenic rat model developing progressive cortical neurofibrillary tangles. Neurobiol Aging 33(7):1448-1456

25. Fitzpatrick AWP et al (2017) Cryo-EM structures of tau filaments from Alzheimer's disease. Nature 547(7662):185-190

26. Frost $B$, Jacks RL, Diamond MI (2009) Propagation of tau misfolding from the outside to the inside of a cell. J Biol Chem 284(19):12845-12852

27. Funk KE et al (2015) Distinct therapeutic mechanisms of tau antibodies: promoting microglial clearance versus blocking neuronal uptake. J Biol Chem 290(35):21652-21662

28. Goedert M, Spillantini MG (2017) Propagation of tau aggregates. Mol Brain 10(1):18

29. Goedert $M$ et al (1996) Assembly of microtubule-associated protein tau into Alzheimer-like filaments induced by sulphated glycosaminoglycans. Nature 383(6600):550-553

30. Golde TE (2014) Open questions for Alzheimer's disease immunotherapy. Alzheimers Res Ther 6(1):3

31. Goni F et al (2018) Anti-beta-sheet conformation monoclonal antibody reduces tau and Abeta oligomer pathology in an Alzheimer's disease model. Alzheimers Res Ther 10(1):10

32. Greenberg SG, Davies P (1990) A preparation of Alzheimer paired helical filaments that displays distinct tau proteins by polyacrylamide gel electrophoresis. Proc Natl Acad Sci U S A 87(15):5827-5831

33. Gu J, Congdon EE, Sigurdsson EM (2013) Two novel tau antibodies targeting the 396/404 region are primarily taken up by neurons and reduce tau protein pathology. J Biol Chem 288(46):33081-33095

34. Guo JL, Lee VM (2014) Cell-to-cell transmission of pathogenic proteins in neurodegenerative diseases. Nat Med 20(2):130-138

35. Guo JL et al (2016) Unique pathological tau conformers from Alzheimer's brains transmit tau pathology in nontransgenic mice. J Exp Med 213(12): 2635-2654

36. Holmes BB, Diamond MI (2014) Prion-like properties of tau protein: the importance of extracellular tau as a therapeutic target. J Biol Chem 289(29): 19855-19861

37. Holmes BB et al (2013) Heparan sulfate proteoglycans mediate internalization and propagation of specific proteopathic seeds. Proc Natl Acad Sci U S A 110(33):E3138-E3147

38. Holmes BB et al (2014) Proteopathic tau seeding predicts tauopathy in vivo. Proc Natl Acad Sci U S A 111(41):E4376-E4385

39. Hrnkova $M$ et al (2007) Neurodegeneration caused by expression of human truncated tau leads to progressive neurobehavioural impairment in transgenic rats. Brain Res 1130(1):206-213 
40. Iba M et al (2013) Synthetic tau fibrils mediate transmission of neurofibrillary tangles in a transgenic mouse model of Alzheimer's-like tauopathy. J Neurosci 33(3):1024-1037

41. Ihse $\mathrm{E}$ et al (2017) Cellular internalization of alpha-synuclein aggregates by cell surface heparan sulfate depends on aggregate conformation and cell type. Sci Rep 7(1):9008

42. Jackson SJ et al (2016) Short fibrils constitute the major species of seedcompetent tau in the brains of mice transgenic for human P301S tau. J Neurosci 36(3):762-772

43. Jadhav $S$ et al (2015) Truncated tau deregulates synaptic markers in rat model for human tauopathy. Front Cell Neurosci 9:24

44. Jadhav S et al (2019) A walk through tau therapeutic strategies. Acta Neuropathol Commun 7(1):22

45. Karch CM, Jeng AT, Goate AM (2012) Extracellular tau levels are influenced by variability in tau that is associated with tauopathies. J Biol Chem 287(51): 42751-42762

46. Katsinelos T et al (2018) Unconventional secretion mediates the transcellular spreading of tau. Cell Rep 23(7):2039-2055

47. Kaufman SK et al (2016) Tau prion strains dictate patterns of cell pathology, progression rate, and regional vulnerability in vivo. Neuron 92(4):796-812

48. Kfoury $\mathrm{N}$ et al (2012) Trans-cellular propagation of tau aggregation by fibrillar species. J Biol Chem 287(23):19440-19451

49. Kontsekova $\mathrm{E}$ et al (2014) Identification of structural determinants on tau protein essential for its pathological function: novel therapeutic target for tau immunotherapy in Alzheimer's disease. Alzheimers Res Ther 6(4):45

50. Kontsekova E et al (2014) First-in-man tau vaccine targeting structural determinants essential for pathological tau-tau interaction reduces tau oligomerisation and neurofibrillary degeneration in an Alzheimer's disease model. Alzheimers Res Ther 6(4):44

51. Kovacech B, Novak M (2010) Tau truncation is a productive posttranslational modification of neurofibrillary degeneration in Alzheimer's disease. Curr Alzheimer Res 7(8):708-716

52. Laurent C, Buee L, Blum D (2018) Tau and neuroinflammation: what impact for Alzheimer's disease and Tauopathies? Biom J 41(1):21-33

53. Levarska $L$ et al (2013) Of rodents and men: the mysterious interneuronal pilgrimage of misfolded protein tau in Alzheimer's disease. J Alzheimers Dis 37(3):569-577

54. Liu CC et al (2016) Neuronal heparan sulfates promote amyloid pathology by modulating brain amyloid-beta clearance and aggregation in Alzheimer's disease. Sci Transl Med 8(332):332ra44

55. Liu L et al (2012) Trans-synaptic spread of tau pathology in vivo. PLoS One 7(2):e31302

56. Macikova I et al (1992) Common and different antigenic properties of the rabies virus glycoprotein of strains SAD-Vnukovo and pitman-Moore. Acta Virol 36(6):541-550

57. Maiza A et al (2018) The role of heparan sulfates in protein aggregation and their potential impact on neurodegeneration. FEBS Lett 592(23):3806-3818

58. Mandelkow EM, Mandelkow E (2012) Biochemistry and cell biology of tau protein in neurofibrillary degeneration. Cold Spring Harb Perspect Med 2(7): a006247

59. Marciniak E et al (2017) Tau deletion promotes brain insulin resistance. J Exp Med 214(8):2257-2269

60. Margittai M, Langen R (2004) Template-assisted filament growth by parallel stacking of tau. Proc Natl Acad Sci U S A 101(28):10278-10283

61. Medina M, Avila J (2014) New perspectives on the role of tau in Alzheimer's disease. Implications for therapy Biochem Pharmacol 88(4):540-547

62. Medina M, Avila J (2014) The role of extracellular tau in the spreading of neurofibrillary pathology. Front Cell Neurosci 8:113

63. Meredith JE Jr et al (2013) Characterization of novel CSF tau and ptau biomarkers for Alzheimer's disease. PLoS One 8(10):e76523

64. Mirbaha $\mathrm{H}$ et al (2015) Tau trimers are the minimal propagation unit spontaneously internalized to seed intracellular aggregation. J Biol Chem 290(24):14893-14903

65. Mudher A et al (2017) What is the evidence that tau pathology spreads through prion-like propagation? Acta Neuropathol Commun 5(1):99

66. Mukrasch MD et al (2005) Sites of tau important for aggregation populate \{beta\}-structure and bind to microtubules and polyanions. J Biol Chem 280(26):24978-24986

67. Nation DA et al (2019) Blood-brain barrier breakdown is an early biomarker of human cognitive dysfunction. Nat Med
68. Nobuhara CK et al (2017) Tau antibody targeting pathological species blocks neuronal uptake and interneuron propagation of tau in vitro. Am J Pathol 187(6):1399-1412

69. Novak M, Kabat J, Wischik CM (1993) Molecular characterization of the minimal protease resistant tau unit of the Alzheimer's disease paired helical filament. EMBO J 12(1):365-370

70. Novak $P$ et al (2017) Safety and immunogenicity of the tau vaccine AADvac1 in patients with Alzheimer's disease: a randomised, double-blind, placebo-controlled, phase 1 trial. Lancet Neurol 16(2):123-134

71. Novak P et al (2018) Ten years of tau-targeted immunotherapy: the path walked and the roads ahead. Front Neurosci 12:798

72. Novak $P$ et al (2018) Tau conformation as a target for disease-modifying therapy: the role of truncation. J Alzheimers Dis 64(s1):S535-S546

73. Novak P et al (2019) AADvac1, an active immunotherapy for Alzheimer's disease and non Alzheimer Tauopathies: an overview of preclinical and clinical development. J Prev Alzheimers Dis 6(1):63-69

74. Rauch JN et al (2018) Tau internalization is regulated by 6-O Sulfation on Heparan sulfate proteoglycans (HSPGs). Sci Rep 8(1):6382

75. Schroeder SK et al (2016) Tau-directed immunotherapy: a promising strategy for treating Alzheimer's disease and other Tauopathies. J Neurolmmune Pharmacol 11(1):9-25

76. Schultz MK Jr et al (2018) Pharmacogenetic neuronal stimulation increases human tau pathology and trans-synaptic spread of tau to distal brain regions in mice. Neurobiol Dis 118:161-176

77. Sebastian-Serrano A, de Diego-Garcia L, Diaz-Hernandez M (2018) The Neurotoxic Role of Extracellular Tau Protein. Int J Mol Sci 19:4

78. Shammas SL et al (2015) A mechanistic model of tau amyloid aggregation based on direct observation of oligomers. Nat Commun 6:7025

79. Shivu B et al (2013) Distinct beta-sheet structure in protein aggregates determined by ATR-FTIR spectroscopy. Biochemistry 52(31):5176-5183

80. Sibille $\mathrm{N}$ et al (2006) Structural impact of heparin binding to full-length tau as studied by NMR spectroscopy. Biochemistry 45(41):12560-12572

81. Sigurdsson EM (2016) Tau immunotherapy. Neurodegener Dis 16(1-2):34-38

82. Skrabana R et al (2004) Folding of Alzheimer's core PHF subunit revealed by monoclonal antibody 423. FEBS Lett 568(1-3):178-182

83. Smolek T et al (2018) First-in-rat study of human Alzheimer's disease tau propagation. Mol Neurobiol

84. Stopschinski BE et al (2018) Specific glycosaminoglycan chain length and sulfation patterns are required for cell uptake of tau versus alphasynuclein and beta-amyloid aggregates. J Biol Chem 293(27):1082610840

85. Sutovsky S et al (2018) Neuropathology and biochemistry of early onset familial Alzheimer's disease caused by presenilin-1 missense mutation Thr116Asn. J Neural Transm (Vienna) 125(6):965-976

86. Takeda $\mathrm{S}$ et al (2015) Neuronal uptake and propagation of a rare phosphorylated high-molecular-weight tau derived from Alzheimer's disease brain. Nat Commun 6:8490

87. Takeda S et al (2016) Seed-competent high-molecular-weight tau species accumulates in the cerebrospinal fluid of Alzheimer's disease mouse model and human patients. Ann Neurol 80(3):355-367

88. Vandermeeren $M$ et al (2018) Anti-tau monoclonal antibodies derived from soluble and filamentous tau show diverse functional properties in vitro and in vivo. J Alzheimers Dis 65(1):265-281

89. von Bergen $\mathrm{M}$ et al (2000) Assembly of tau protein into Alzheimer paired helical filaments depends on a local sequence motif ((306) VOIVYK (311)) forming beta structure. Proc Natl Acad Sci U S A 97(10):5129-5134

90. von Bergen $\mathrm{M}$ et al (2001) Mutations of tau protein in frontotemporal dementia promote aggregation of paired helical filaments by enhancing local beta-structure. J Biol Chem 276(51):48165-48174

91. Wang YP et al (2007) Stepwise proteolysis liberates tau fragments that nucleate the Alzheimer-like aggregation of full-length tau in a neuronal cell model. Proc Natl Acad Sci U S A 104(24):10252-10257

92. Wischik CM et al (1988) Structural characterization of the core of the paired helical filament of Alzheimer disease. Proc Natl Acad Sci U S A 85(13):4884-4888

93. Wischik CM et al (1988) Isolation of a fragment of tau derived from the core of the paired helical filament of Alzheimer disease. Proc Natl Acad Sci U S A 85(12):4506-4510

94. Wischik CM et al (1996) Selective inhibition of Alzheimer disease-like tau aggregation by phenothiazines. Proc Natl Acad Sci U S A 93(20):1121311218 
95. Wu JW et al (2013) Small misfolded tau species are internalized via bulk endocytosis and anterogradely and retrogradely transported in neurons. Biol Chem 288(3):1856-1870

96. Yamada K (2017) Extracellular tau and its potential role in the propagation of tau pathology. Front Neurosci 11:667

97. Yamada K (2017) In vivo microdialysis of brain interstitial fluid for the determination of extracellular tau levels. Methods Mol Biol 1523:285-296

98. Yamada $\mathrm{K}$ et al (2011) In vivo microdialysis reveals age-dependent decrease of brain interstitial fluid tau levels in P301S human tau transgenic mice. J Neurosci 31(37):13110-13117

99. Yamada K et al (2014) Neuronal activity regulates extracellular tau in vivo. J Exp Med 211(3):387-393

100. Yamada K et al (2015) Analysis of in vivo turnover of tau in a mouse model of tauopathy. Mol Neurodegener 10:55

101. Yamada M, Hamaguchi T (2018) The sulfation code for propagation of neurodegeneration. J Biol Chem 293(27):10841-10842

102. Yanamandra K et al (2013) Anti-tau antibodies that block tau aggregate seeding in vitro markedly decrease pathology and improve cognition in vivo. Neuron 80(2):402-414

103. Zhao J et al (2017) Glycan determinants of heparin-tau interaction. Biophys J 112(5):921-932

104. Zhou Y et al (2018) Relevance of phosphorylation and truncation of tau to the Etiopathogenesis of Alzheimer's disease. Front Aging Neurosci 10:27

105. Zilka N et al (2012) Who fans the flames of Alzheimer's disease brains? Misfolded tau on the crossroad of neurodegenerative and inflammatory pathways. J Neuroinflammation 9:47

106. Zilka N et al (2012) The self-perpetuating tau truncation circle. Biochem Soc Trans 40(4):681-686

107. Zimova I et al (2016) Human truncated tau induces mature neurofibrillary pathology in a mouse model of human Tauopathy. J Alzheimers Dis 54(2): 831-843

\section{Publisher's Note}

Springer Nature remains neutral with regard to jurisdictional claims in published maps and institutional affiliations.

Ready to submit your research? Choose BMC and benefit from:

- fast, convenient online submission

- thorough peer review by experienced researchers in your field

- rapid publication on acceptance

- support for research data, including large and complex data types

- gold Open Access which fosters wider collaboration and increased citations

- maximum visibility for your research: over $100 \mathrm{M}$ website views per year

At $\mathrm{BMC}$, research is always in progress.

Learn more biomedcentral.com/submissions 